\begin{tabular}{|c|l|}
\hline Title & Survival analysis on bridges for modeling bridge replacement and eval uating bridge performance \\
\hline Author(s) & Beng, Si Soon; Matsumoto, Takashi \\
\hline Citation & $\begin{array}{l}\text { Structure and Infrastructure Engineering, 8(3), 251-268 } \\
\text { https://doi.org/10.1080/15732471003594393 }\end{array}$ \\
\hline Issue Date & 2012-03 \\
\hline Doc URL & http://hdl.handle.net/2115/50708 \\
\hline Type & article \\
\hline File Information & SIE_8_251-268.pdf \\
\hline
\end{tabular}

Instructions for use 
Abbreviated version of title: Survival analysis on bridges

\title{
Survival Analysis on Bridges for Modeling Bridge Replacement and Evaluating Bridge Performance
}

\author{
Si Soon Beng $\dagger$ and Takashi Matsumoto ${ }^{*} \ddagger$ \\ $\dagger$ M.S.E., Dept. of Civil Eng., Univ. of Tokyo (7-3-1, Hongo, Bunkyo-ku, Tokyo 113-8656, Japan) \\ soonbeng78@gmail.com \\ * + Ph.D., Assoc. Prof., Div. of Built Environment, Hokkaido Univ. (Kita 13, Nishi 8, Kita-ku, \\ Sapporo 060-8628, Japan, corresponding author) takashim@eng.hokudai.ac.jp
}

\begin{abstract}
A sustainable civil infrastructure is a prerequisite for achieving sustainability in infrastructure development. However, the finite lifetime of infrastructure indicates a latent mass-maintenance problem in some countries which had experienced mass-construction of infrastructure during high economic growth. To achieve optimal infrastructure management for overcoming this mass-maintenance problem, life prediction of infrastructure is indispensable. Hence, in this research, bridge life assessment from actual bridge survival data of U.S. and Japan was carried out by using the method of survival analysis, a method commonly applied in the field of epidemiology for the analysis of lifetime. The results estimated average bridge life at 93.5 years and a bridge replacement projection model was successfully derived. The bridge replacement projection model can be applied as an approximation for other countries where the same design specifications are followed. By carrying out stratifications of bridge cohorts in U.S., evaluations of bridge performance with respect to structure length; traffic volume and truck traffic volume were also executed.
\end{abstract}

Keywords: Survival analysis; Cohort; Kaplan-Meier survival curve; Bridge replacement projection model; Stratification 


\section{Introduction}

A sound infrastructure system has long been considered an important prerequisite for achieving economic development. Realizing the strong link between infrastructure development and economic growth, many countries, in the process of chasing after high economic growth, invested heavily in infrastructure stocks.

However, as infrastructure stocks accumulate and aging takes place, and if insufficient is done to maintain these infrastructure at an appropriate condition, infrastructure system collapses are likely to occur, which will in turn bring about negative impacts to the economy. Already, some developed countries such as the United States and Japan had experienced or are beginning to feel the gravity of the problem. In the United States, for instance, infrastructure system failure in New York during the 1980 s and the numerous large-scaled blackouts which occurred in recent years were the consequences of massive infrastructure stocks left with negligent care and constrictive budgets. For the case of Japan, deterioration problems of the huge amount of infrastructure constructed during the high economic growth period (1955 1972) are perplexing many of the infrastructure owners.

On the other hand, in the past few decades, Asian developing countries, especially those in the East Asian region, have sustained an impressive growth rate. The growth pattern was so high and unprecedented in the history of economic development that World Bank interpreted it as the 'East Asian Miracle'. And in the process of rapid economic growth, mass-construction of infrastructure 
occurred in these East Asian countries.

Mass-construction of infrastructure systems at current times implies mass-maintenance of these infrastructure systems in future. As a future possibility, these East Asian countries might be faced with the mass-maintenance problem of infrastructures in time to come. Under the threat of such a potential crisis, it is essential to be able to predict the time and scale of mass-maintenance for each of these countries, so that awareness can be raised and preventive measures be adopted at an early stage. All these measures will help to contribute towards a sustainable infrastructure system in these countries for the future.

Faced with the difficulty of having to manage a deteriorating infrastructure system under a limited budget, many infrastructure owners (especially those in the developed countries) have begun projections on future maintenance and replacement needs for infrastructure stocks which are under their administration, so as to achieve optimal infrastructure management. However, since not much is known about the physical lifespan of infrastructure such as bridges, rough estimates based on depreciation service lives are used instead. Table 1 shows depreciation service lives for some categories of infrastructure which are set by the Ministry of Finance in Japan (MOF 1998). However, problem arises from using depreciation service life to represent replacement cycle since the former is set from an economic point of view and does not reflect real physical lifespan of an infrastructure which is the key factor for decision making on replacement. 
Hence, in this research, although there are many infrastructure categories, attention will only be focused on 'bridges'. And for this category of infrastructure, the main objectives are listed as below:

(i) To assess bridge service life and at the same time derive a bridge replacement projection model.

(ii) To evaluate bridge performance with respect to variables such as structural dimension and traffic intensity.

\section{Methodology used in the derivation of a bridge replacement projection model}

In the derivation of a bridge replacement projection model, bio-statistical methods which are commonly used in the field of epidemiology are used (Newman 2001). These statistical methods, for the analysis of lifetime or response time, are more suited to handle survival data than standard statistical procedures used in data analysis since survival data has the special feature of being not symmetrically distributed. An additional feature of survival data which renders standard methods inappropriate is that survival times are frequently censored.

The methods for analyzing survival data are not restricted to the field of epidemiology. The methodology had also been extended to the field of engineering such as in the analysis of the lifetimes of industrial or electronic components (Kalbfleisch and Prentice 1980, Lawless 2003). We go a step further and make an attempt to apply this methodology in the field of Civil Engineering, by using it in the lifetime analysis of bridges. Here, analogies are drawn between a human being and a bridge as the subject, and between the death of a human being and the replacement of a bridge as the 
endpoint of interest in our study.

\subsection{Survival analysis}

In the course of medical research, data on the time to the occurrence of a particular event, such as the death of a patient, are frequently encountered. Such data are generically referred to as survival data. However, the event of interest need not necessarily be death, but could, for example, be the end of a period spent in remission from a disease, relief from symptoms, or the recurrence of a particular condition. General methods for analyzing survival data are available and these methods are referred to collectively as survival analysis (Klein and Moeschberger 1997).

Survival analysis is used for analyzing cohort data and although there are many types of cohort studies, they can be broadly classified into two types, namely closed cohort study and open cohort study. In a closed cohort study, subjects either develop the disease or not, and all those not developing it necessarily have the same length of follow-up, namely, the maximum observation time. However, in reality of cohort studies, subjects may cease to be under observation prior to either developing the disease or reaching the end of the planned period of follow-up. This can occur for a variety of reasons, such as the subject being lost to follow-up by the investigator, the subject deciding to withdraw from the study, or the investigator eliminating the subject from further observation due to the development of an inter-current condition which conflicts with the aims of the study. Cohort studies in which subjects have different maximum observation times are referred to as 
open cohort studies.

For historical reasons, it is usual in the survival analysis literature to refer to the study endpoint as "death" and to the length of follow-up for a given subject as the "survival" time. These and related conventions are adopted irrespective of whether the study has a mortality endpoint or not. In the statistical theory of survival analysis, survival time is regarded as a continuous random variable that we denote by $T$. As with any continuous random variable, $T$ has an associated probability function $f(t)$. In survival analysis it is generally more convenient to characterize $T$ in terms of two other functions, namely, the survival function $S(t)$ and the hazard function $h(t)$.

The survival function $S(t)$ is defined to be the probability that the survival time is greater than or equal to $t$, and so

$$
S(t)=P(T \geq t)=1-F(t)
$$

where $F(t)$ is the cumulative density function and is given by

$$
F(t)=P(T<t)=\int_{0}^{t} f(u) d u .
$$

The survival function can therefore be used to represent the probability that an individual survives from the time origin to some time beyond $t$.

The hazard function is the probability that an individual dies at time $t$, conditional on he or she having survived to that time. The hazard function therefore represents the instantaneous death rate for an individual surviving to time $t$. To obtain a more formal definition of the hazard function, 
consider the probability that the random variable associated with an individual's survival time, $T$, lies between $t$ and $t+\delta t$, conditional on $T$ being greater than or equal to $t$, written $P(t \leq T<t+\delta t \mid T$ $\geq t$ ). The hazard function $h(t)$ is then the limiting value of this probability divided by the time interval $\delta t$, as $\delta t$ tends to zero, so that

$$
h(t)=\lim _{\delta t \rightarrow 0}\left\{\frac{P(t \leq T \leq t+\delta t \mid T \geq t)}{\delta t}\right\} .
$$

A useful identity relating the three functions; survival function, probability function and hazard function is given as in equation (4).

$$
h(t)=\frac{f(t)}{S(t)} .
$$

In the analysis of survival data, the survival function and hazard function will be estimated from the observed survival times. The probability function can then be derived simply by applying equation (4).

Since $T$ is a continuous random variable, the probability of dying at any given time is 0 . It is only when we consider the probability of dying in an interval of time that a nonzero probability is obtained. For this reason, we sometimes refer to $f(t)$ and $h(t)$ as "instantaneous" probabilities. In summary, we can characterize $S(t), f(t)$ and $h(t)$ as follows: $S(t)$ is the probability that an individual alive at time 0 will survive to time $t ; f(t)$ is the instantaneous probability per unit time that an individual alive at time 0 will die at time $t$; and $h(t)$ is the instantaneous probability per unit time that an individual alive at time $t$ will die "in the next instant". 
In order to give a clearer picture of the implicit meaning of each of these functions, the survival, probability and hazard functions for a hypothetical human cohort, in which a large group of newborns is followed until the last subject dies, are illustrated in Figure 1. Note that Figure 1(a) and (b) have been truncated at age 100 years, and Figure 1(c) at age 70 years.

In Figure 1(a), the survival function is non-increasing and ultimately decreases to 0 at the upper limit of life length. Although somewhat difficult to appreciate from the graph, there is a sharp drop in the survival curve in the first year of life due to prenatal and other causes of death following the newborn period. After that there is a very gradual decline until late middle age, and then a precipitous drop as old age approaches.

In Figure 1(b), the probability function reflects the same phenomena in that there is a steep slope in the first year of life, a gentle increase throughout late middle age, and then a large peak in the curve in the senior years. The area under the curve between any two ages equals the probability of dying in that age interval. This explains why so much of the area is concentrated at older ages. The curve declines rapidly as 100 years is approached because very few members of the cohort survive long enough to die at such old age.

In Figure 1(c), the hazard function demonstrates the same patterns observed in the survival function and probability function. In particular, we note the rapid increase in the hazard function as extreme old age approaches. This shows that for someone who has lived to be very old, the risk of 
dying in the next instant gets progressively greater as time passes.

\subsection{Kaplan-Meier method for censored survival data}

The Kaplan-Meier method is often used to estimate a survival function from censored survival data. An important feature of this method is that nothing is assumed about the functional form of either the survival curve or the hazard function.

Consider an open cohort study involving $r$ subjects and let the observations be $t_{1}, t_{2}, \ldots, t_{r}$. We assume that $t_{i}>0$ for all $i$, which ensures that each member of the cohort is followed for at least a small amount of time. Now, suppose that among the $r$ survival times there are $J$ death times: $\tau_{1}<\cdots<$ $\tau_{j}<\cdots<\tau_{J}$. Let $\tau_{0}=0$ and denote the maximum survival time by $\tau_{J+1}$, that is $\tau_{J+1}=\max \left(t_{1}, t_{2}, \ldots, t_{r}\right)$. The Kaplan-Meier approach to censored survival data begins by partitioning the period of follow-up into $J+1$ intervals using the death times as cutpoints: $\left[\tau_{0}, \tau_{1}\right),\left[\tau_{1}, \tau_{2}\right), \ldots,\left[\tau_{j}, \tau_{j+1}\right), \ldots,\left[\tau_{J-1}, \tau_{J}\right),\left[\tau_{J}, \tau_{J}\right.$ $\left.{ }_{+1}\right]$, where we note that the last interval contains $\tau_{J+1}$. We refer to $\left[\tau_{j}, \tau_{j+1}\right)$ as the $j$ th interval. In many applications, there will be considerable censoring at $\tau_{J+1}$ due to subjects surviving to the end of the study. If $\tau_{J+1}$ is a death time, then $\tau_{J}=\tau_{J+1}$ and the last interval shrinks to the single point $\tau_{J}$. Let $a_{j}$ be the number of deaths at $\tau_{j}$ and let $c_{j}$ be the number of censored observations in the $j$ th interval $(j=$ $0,1, \ldots, J)$. By definition, $a_{0}=0$.

The group of subjects "at risk" at $\tau_{j}$, also referred to as the $j$ th risk set, consists of those individuals with a survival time greater than or equal to $\tau_{j}(j=0,1, \ldots, J)$. In other words, the $j$ th risk set consists 
of all subjects who are alive just prior to $\tau_{j}$.

Let $r_{j}$ denote the number of subjects in the $j$ th risk set $(j=0,1, \ldots, J)$, and denote by $r_{J+1}$ the number of subjects who survive to $\tau_{J+1}$. Since subjects exit the cohort only by death or censoring, it follows that

$$
r_{j+1}=r_{j}-a_{j}-c_{j}
$$

$(j=0,1, \ldots, J)$.

Equation (5) derived above applies to an open cohort study. In our application of the survival analysis theory to bridges, we are more concerned with a closed cohort study i.e. no censoring occurs except at $\tau_{J+1}$. In this case, $c_{j}=0(j=0,1, \ldots, J)$, and substituting this attribute into equation (5), we obtain

$$
r_{j+1}=r_{j}-a_{j}
$$

We will now derive an estimate of the survival function $S(t)$ based on the above partition and certain conditional probabilities. For brevity we denote $S\left(\tau_{j}\right)$ by $S_{j}$ so that, in particular, $S_{0}=1$. For $j>0$, consider the interval $\left[\tau_{j}-\varepsilon, \tau_{j}+\varepsilon\right]$, where $\varepsilon$ is a positive number that is small enough to ensure that the interval does not contain any death times other than $\tau_{j}$ or any censoring times. For $j=0$, we use the interval $\left[\tau_{0}, \tau_{0}+\varepsilon\right]$ instead. Let $p_{j}$ denote the conditional probability of surviving to $\tau_{j}+\varepsilon$, given survival to $\tau_{j}-\varepsilon(j=0,1, \ldots, J)$. That is, $p_{j}$ is the conditional probability of surviving to just after $\tau_{j}$, given survival to just prior to $\tau_{j}$. Let $q_{j}=1-p_{j}$ be the corresponding conditional probability 
of dying. Since there are $r_{j}$ subjects at risk at $\tau_{j}-\varepsilon$, and $a_{j}$ of them die prior to $\tau_{j}+\varepsilon$, the binomial estimates are

$$
\hat{q}_{j}=\frac{a_{j}}{r_{j}}
$$

and

$$
\hat{p}_{j}=1-\hat{q}_{j}=\frac{r_{j}-a_{j}}{r_{j}}
$$

$(j=0,1, \ldots, J)$. These estimates are valid regardless of how small we take $\varepsilon$. Since $a_{0}=0$, it follows that $\hat{q}_{0}=0$ and $\hat{p}_{0}=1$.

The probability of surviving from $\tau_{0}$ to $\tau_{1}$ is $p_{1}$. For those who survive to $\tau_{1}$, the conditional probability of surviving to $\tau_{2}$ is $S_{2}=p_{1} p_{2}$. Likewise, the probability of surviving from $\tau_{0}$ to $\tau_{3}$ is $S_{2}=p_{1} p_{2} p_{3}$. Proceeding in this way we obtain the probability $S_{j}=p_{1} p_{2} \cdots p_{j}$ of surviving from $\tau_{0}$ to $\tau_{j}(j=0,1, \ldots, J)$. The Kaplan-Meier estimate of $S_{j}$ is

$$
\hat{S}_{j}=\hat{p}_{1} \hat{p}_{2} \cdots \hat{p}_{j}
$$

$(j=0,1, \ldots, J)$. We define $\hat{S}_{0}=1$ and $\hat{S}_{J+1}=\hat{S}_{J}$. Since there are no deaths in the $j$ th interval other than at $\tau_{j}, \hat{S}(t)$ equals $\hat{S}_{j}$ for all $t$ in the interval, and so the graph of $\hat{S}(t)$ is a step function.

When the Kaplan-Meier estimate of the survival function is graphed as a function of time, it will be referred to as the Kaplan-Meier survival curve. A schematic representation of a Kaplan-Meier survival curve is shown in Figure 2. Note that each of the line segments making up the steps includes 
the left endpoint (indicated by a solid dot) but not the right endpoint (indicated by a circle), except for the final line segment that includes both endpoints. This is consistent with the way intervals were defined. If $\tau_{J+1}$ is a death time, the final line segment shrinks to a single point. To enhance visual appearance, it is common to join the steps with vertical lines in the illustration of the Kaplan-Meier survival curve.

Usually, one of the aims of a survival analysis is to determine whether a given exposure is related to survival. One approach is to stratify the cohort according to exposure categories and compare the resulting Kaplan-Meier survival curves. For simplicity, suppose that exposure is dichotomous and that, at every death time, the survival curve for the exposed cohort lies below that for the unexposed cohort. Figure 3 shows a graphical depiction of such a cohort.

A finding such as this suggests that exposure is associated with a decrease in survival. The question then arises as to how the observed difference in survival should be measured. To quantify the extent of observed differences, hypothesis testing procedures such as the log-rank test and the Wilcoxon test can be utilized (Collett 1994).

\subsection{Modeling survival data by using Weibull distribution}

The Kaplan-Meier method is based on relatively few assumptions; in particular, nothing is specified regarding the functional form of either the survival function or the hazard function. It is appropriate to view a Kaplan-Meier survival curve as a scatter plot of censored survival data. The 
appearance of a Kaplan-Meier curve can be used to form ideas about the nature of the underlying survival function and hazard function, in much the same way as a scatter plot is used as a visual aid in linear regression.

Despite these advantages, there are difficulties with the Kaplan-Meier approach. Kaplan-Meier curves are not designed to smooth the data while accounting for random variation in the way that a linear regression line is fitted to points in a scatter plot. Hence, parametric modeling of survival data, based on the Weibull distribution, is essential.

The Weibull distribution, introduced by W. Weibull in 1951 in the context of industrial reliability testing, is a probability distribution which plays a central role in the analysis of survival data. Indeed, this distribution is as central to the parametric analysis of survival data as the normal distribution is in linear modeling.

The Weibull distribution has the survival function $S(t)$ given by

$$
S(t)=\exp \left(-\lambda t^{\alpha}\right)
$$

and hazard function $h(t)$ given by

$$
h(t)=\alpha \lambda t^{\alpha-1} .
$$

According to the identity described in equation (4), the corresponding probability function $f(t)$ is then given by

$$
f(t)=\alpha \lambda t^{\alpha-1} \exp \left(-\lambda t^{\alpha}\right)
$$


Here, $\lambda$ and $\alpha$ are parameters satisfying the conditions $\lambda>0$ and $\alpha>0$. We refer to $\lambda$ as scale parameter and to $\alpha$ as shape parameter.

Parametric models, which include the Weibull distribution, are characterized by parameters that in practice, are usually unknown. To estimate such parameters from survival data, the maximum likelihood method is a method which is commonly used. The maximum likelihood estimates of these parameters are those values for which the likelihood function is a maximum. In a usual situation where the survival data include one or more censored survival times, the total likelihood function can be expressed as

$$
\prod_{j=1}^{r} f\left(t_{j}\right) \prod_{l=1}^{n-r} S\left(t_{l}\right)
$$

in which the first product is taken over the $r$ death times and the second over the $n-r$ censored survival times.

More compactly, suppose that the data are regarded as $n$ pairs of observations, where the pair for the $i$ 'th individual is $\left(t_{i}, \delta_{i}\right), i=1,2, \ldots, n$. In this notation, $\delta_{i}$ is an indicator variable which takes the value zero when the survival time $t_{i}$ is censored and unity when $t_{i}$ is an uncensored survival time.

The likelihood function can then be expressed in the form

$$
\prod_{i=1}^{n}\left\{f\left(t_{i}\right)\right\}^{\delta_{i}}\left\{S\left(t_{i}\right)\right\}^{1-\delta_{i}} .
$$

This function, which is equivalent to that in equation (13), can then be maximized with respect to the unknown parameters in the probability and survival functions. 
In the case of a Weibull distribution, substituting the probability and survival functions according to equations (10) and (12), the likelihood function is expressed as

$$
\prod_{i=1}^{n}\left\{\alpha \lambda t_{i}^{\alpha-1} \exp \left(-\lambda t_{i}^{\alpha}\right)\right\}^{\delta_{i}}\left\{\exp \left(-\lambda t_{i}^{\alpha}\right)\right\}^{1-\delta_{i}}
$$

where $\delta_{i}$ is zero if the $i^{\prime}$ th survival time is censored and unity otherwise. This is regarded as a function of $\lambda$ and $\alpha$, the unknown parameters in the Weibull distribution, and so can be written $L(\lambda$, $\alpha$ ). The corresponding log-likelihood function is given by

$$
\log L(\lambda, \alpha)=\sum_{i=1}^{n} \delta_{i} \log (\alpha \lambda)+(\alpha-1) \sum_{i=1}^{n} \delta_{i} \log t_{i}-\lambda \sum_{i=1}^{n} t_{i}^{\alpha},
$$

and noting that

$$
\sum_{i=1}^{n} \delta_{i}=r
$$

the log-likelihood becomes

$$
\log L(\lambda, \alpha)=r \log (\alpha \lambda)+(\alpha-1) \sum_{i=1}^{n} \delta_{i} \log t_{i}-\lambda \sum_{i=1}^{n}{t_{i}}^{\alpha}
$$

The maximum likelihood estimates of $\lambda$ and $\alpha$ are found by differentiating this function with respect to $\lambda$ and $\alpha$, equating the derivatives to zero, and evaluating them at $\hat{\lambda}$ and $\hat{\alpha}$. The resulting equations are

$$
\frac{r}{\hat{\lambda}}-\sum_{i=1}^{n} t_{i}^{\hat{\alpha}}=0
$$

and

$$
\frac{r}{\hat{\alpha}}+\sum_{i=1}^{n} \delta_{i} \log t_{i}-\hat{\lambda} \sum_{i=1}^{n} t_{i}^{\hat{\alpha}} \log t_{i}=0
$$

From equation (15), 


$$
\hat{\lambda}=r / \sum_{i=1}^{n} t_{i}^{\hat{\alpha}}
$$

and on substituting for $\hat{\lambda}$ in equation (16), we get the equation

$$
\frac{r}{\hat{\alpha}}+\sum_{i=1}^{n} \delta_{i} \log t_{i}-\frac{r}{\sum_{i=1}^{n} t_{i}^{\hat{\alpha}}} \sum_{i=1}^{n} t_{i}^{\hat{\alpha}} \log t_{i}=0 .
$$

This is a non-linear equation in $\hat{\alpha}$ which can only be solved using an iterative numerical procedure.

Once the estimate $\hat{\alpha}$ which satisfies equation (18) has been found, equation (17) can be used to obtain $\hat{\lambda}$.

To solve the non-linear equation given in equation (18), computer software is required. Fortunately, some of the widely used statistical packages such as SAS, S-PLUS, BMDP and SPSS are well-equipped to handle survival analysis problems. In this research, S-PLUS will be used to obtain the maximum likelihood estimates for the unknown parameters of the Weibull distribution i.e. $\lambda$ and $\alpha$.

\section{Derivation of a bridge replacement projection model}

Utilizing detailed bridge construction and replacement data for two developed countries; namely, Japan and the United States, and by conducting survival analysis on the bridges in these two countries, we were able to derive a bridge replacement projection model as well as predict the mean service life of bridges. 


\subsection{Survival analysis on bridges in Japan}

As the first step in the derivation of a bridge replacement projection model, we performed survival analysis on bridges in Japan. Here, 'bridges' are defined as national highway and prefectural road bridges with spans of more than $15 \mathrm{~m}$. Such a constriction on the definition of bridges arises because the bridge replacement data available are only for bridges with such classification.

Past actual bridge replacement records of Japan for the period 1967 1996 can be found in three investigation reports which were compiled by the Public Works Research Institute of the ex Ministry of Construction (PWRI, 1977, 1986, 1996). The investigation on replacement circumstances of existing bridge stock is conducted in a 10-year interval and so far, three investigations had been conducted in 1977, 1986 and 1996. Each investigation report covered a span of ten years and examined the reasons for replacement as well as the service lives of bridges, with the aims of improving the durability of bridges and serving as the basis in life-cycle design.

In order to conduct survival analysis on the bridges in Japan, having past replacement records alone is insufficient. In addition, we need information on the number of subjects in each cohort i.e. the number of newly constructed bridges in each year. Fortunately, this source of information is available through the Highway Statistical Yearbook (JHUC, 1964 2003). In the yearbooks, bridges are classified by function and span and thus, it is possible to pick out only those that are national highway and prefectural road bridges with spans of more than $15 \mathrm{~m}$. 
Since replacement data is only available for the period 1967 1996, any survival analysis performed is only feasible for cohort 1967 and beyond. Under such data constraint, we performed survival analysis for bridge cohorts of 1968 and beyond for the case of Japan. The resulting Kaplan-Meier survival curves for the five cohorts of 1968 1972 are plotted and shown in Figure 4.

From the figure, it can be observed that all the five cohorts displayed similar Kaplan-Meier survival curves. An average Kaplan-Meier survival curve is plotted for the five cohorts of 1968 1972, and this five-year average curve is represented by the curve in bold shown in Figure 4. For simplicity, we will let this five-year average curve to be the representative Kaplan-Meier survival curve for bridges in Japan. In section 3.3, this representative Kaplan-Meier survival curve for Japan will be held in comparison with Kaplan-Meier survival curves for bridges in the United States as a mean to determine differences in bridge performance between the two countries.

\subsection{Survival analysis on bridges in the United States}

The next step was to analyze the situation of bridge replacement in a different country. In this case, we chose the United States as our target for studies since a complete and thorough database on bridges in the United States is available through the National Bridge Inventory (NBI) which had been prepared by Federal Highway Administration (FHWA) of the U.S. Department of Transportation (FHWA, 2003a, b).

The resulting Kaplan-Meier survival curves for some cohorts of bridges are shown in Figure 5. 
What is conspicuous is the difference in behaviors between the pre-1930 cohorts and the post-1930

cohorts. The pre-1930 cohorts (i.e. cohorts 1900, 1910 and 1920) displayed high durability in the early periods but encountered a steep drop in survivability in recent history. On the other hand, the post-1930 cohorts (i.e. cohort 1930 and beyond) exhibited similar bridge performances, although a slight improvement can be observed in the youngest cohorts of 1970 and 1980.

To explain the behavior displayed by the pre-1930 cohorts, we will start off by explaining the two principal reasons for bridge replacement. Bridge replacements can be due to many reasons but these reasons can be generally classified under the following two principal reasons; namely, structural inadequacy and functional inadequacy (OECD, 1992).

-- Structural inadequacy i.e. service closure due to failures, with the bridge becoming unsafe due to insufficient structural stability, a common cause of failure for old bridges. This corresponds to the "useful" life or "structural" life of a bridge which can be very long, for example 50 to 100 years or more, if there is no overload, collision or natural disaster. Some of the sub-factors under this category include damages due to environmental actions (salt, frost) and traffic on the bridge, too many elements to replace, catastrophic failure (collapse) and etc.

-- Functional inadequacy i.e. service closure of a still structurally sound bridge whose loading capacity or geometry, for instance, does not meet actual traffic requirements. This "functional" life is less than the structural life, for example, in countries with high economic growth. Some of the 
sub-factors under this category include replacements due to new standard, road/canal widening, bottlenecks in loads and clearance, seismic retrofitting and etc.

Therefore, a possible explanation for the behavior shown in, say for example, cohort 1900 is that in the early stage, traffic requirements were low and bridge replacements were mainly due to structural inadequacy. And since traffic volume was low at that time, not many bridges failed due to structural inadequacy, causing a gentle drop in the survival curve. However, as industrialization took place and traffic requirements increased, the loading capacities or geometries of most bridges in cohort 1900 failed to meet actual traffic requirements. As a result, in the later stage, many bridges in this cohort were replaced due to functional inadequacy, causing a steep drop in the survival curve.

Notice that in Figure 5, flattening in the steepness of the Kaplan-Meier survival curves at the end portion, against expectation, can be observed for almost every cohort. The plateau spans for a period of about 12 years in each cohort, suggesting that for the past decade (1990 2002), actual replacements carried out fell short of what it was expected to be. Two possible explanations for such a phenomenon are that 1) a shortage in funds constrained the number of bridges which could be replaced since new construction of bridges had to take place concurrently; and 2) there might be a shift in bridge management policy from one which is of build-and-scrap nature to a more maintenance oriented one, thus prolonging the life spans of the bridges.

Fitting a Weibull distribution directly to a Kaplan-Meier survival curve with flattening of 
steepness at the end portion will tend to produce a more optimistic projection model than one which chooses to neglect the plateau portion. To adopt a more conservative stance, we will ignore the last 12 years of the Kaplan-Meier survival curve when performing Weibull model fitting to the curve.

\subsection{Comparison of Kaplan-Meier survival curves between Japan and the United States}

The resulting Kaplan-Meier survival curves for both countries: Japan and the United States are then compared in order to gauge the difference in bridge performance between the two countries (see Figure 6). Since the representative curve for Japan is obtained by taking the average of the survival curves for cohorts 1968 1972, it should be held in comparison with cohorts of the United States from the same era. That is the reason for selecting cohorts 1960 and 1970 of the United States as the subjects for comparison.

From Figure 6, interestingly, we can observe that there is a very good correspondence between the representative curve for Japan and the survival curve for cohort 1960 of the United States. This goes to show that bridge performance between Japan and the United States is very much alike, at least for the initial twenty-four years (as depicted in the graph), despite all the differences in social and geographical conditions between the two countries.

As explained earlier, the representative survival curve for Japan and the survival curve for cohort 1960 of the United States corresponded well with each other. From Figure 5, we can observe that the Kaplan-Meier survival curves for cohorts 1930 1960 agree well with each other. Hence, from these 
two observations, we can assume cohort 1930 as the representative cohort for the United States in order to get a longer time period, and fit a Weibull distribution to the survival curve of this cohort so as to obtain a suitable projection model for bridge replacement.

So far, we had shown that Japan and the United States displayed similar bridge performances. And since many countries follow either the Japanese or the American specifications in the design of their bridges, we can rightfully claim that the bridge replacement projection model which we will obtain for the United States in the following section could be applicable for these countries as an approximation.

\subsection{Weibull model fitting and derivation of a bridge replacement projection model}

A Weibull model is then fitted into this representative Kaplan-Meier survival curve of the United States (i.e. survival curve for cohort 1930 shown in Figure 5), using the statistical package S-PLUS. As mentioned in section 3.2 , to adopt a more conservative stance, the last 12 years of the Kaplan-Meier survival curve will be ignored when fitting the Weibull model to the curve.

As shown in Figure 7, the Weibull model provided a reasonably good fit to the Kaplan-Meier survival curve for the representative cohort of the United States. Based on this Weibull model, the maximum likelihood estimates for the unknown parameters $\lambda$ and $\alpha$ are given by $\lambda=9.476 \times 10^{-3}$ and $\alpha=2.169$.

Once we know the values of the two unknown parameters $\lambda$ and $\alpha$, we can plot the survival 
function, probability function and hazard function by using the formulas given in equations (12) (14). The plots for the survival function, probability function and hazard function are illustrated in Figure 8(a), (b) and (c), respectively.

According to the survival function illustrated in Figure 8(a), it will take approximately 230 years to replace all bridges in a cohort. Of course, there is much to debate on the reliability of this survival function since only the first 60 years of the curve is derived from actual data (as represented by the bolded line), while the major part of the curve is only a result of extrapolation (as represented by the fainted line). However, this problem will always occur for service life prediction of bridges since the lifespan of a bridge is too long to allow follow-up until the last bridge in a cohort is replaced.

From the probability function given in Figure 8(b), we can obtain an estimate of the mean service life of a bridge by applying fundamental statistical knowledge. Here, the mean or expected value for the service life of a bridge can be calculated from the probability function by using the following attribute

$$
E(t)=\int_{0}^{\infty} t f(t) d t
$$

where $E(t)$ is the mean or expected value, and $f(t)$ is the probability function. Using this attribute, the mean service life of a bridge is calculated to be 93.5 years, which is significantly longer than the depreciation service life of 60 years set by the Ministry of Finance in Japan, but remains a reasonable figure when compared to speculations of a hundred years as noted in some literatures. 
Now, this probability function will be our bridge replacement projection model, or so called 'proposed projection model', since multiplying the probability per unit time at any time $t$ by the number of newly constructed bridges at time 0 will give us the number of bridges in that cohort that will 'die' at time $t$.

In Figure 8(c), it can be seen that the hazard function is a monotonously increasing function, implying that the risk of a bridge getting replaced in the following year gets progressively greater as the bridge ages.

\subsection{Verification of proposed projection model}

Figure 9 shows the number of bridges built yearly in the United States for the period 1900 2002.

From the figure, we can observe that construction boom in the United States occurred in two periods, namely the 1930s and the 1960s. For the past decade, we can also notice a steady drop in the number of new constructions in the United States.

By applying the proposed bridge replacement projection model to this construction data, estimates of bridge replacements can be obtained. Hence, to verify the proposed projection model, we shall compare actual replacements in the United States with the projection results obtained by using the proposed projection model for the period 1900 2002. The results are shown in Figure 10. In the same graph, the projection results obtained by using conventional methods 1 and 2 are also plotted in order to hold comparisons with the result of the proposed projection model. 
Here, conventional method 1 refers to the projection method when assuming a bridge generation cycle of 60 years. On the other hand, conventional method 2 refers to the projection method when assuming a Gamma distribution with a mean service life of 60 years.

From Figure 10, it is very obvious that the projection results obtained by using both conventional methods differed greatly from the actual replacements. In contrast, the proposed projection model offered a much more accurate projection result. In fact, the proposed projection model can be considered to be very accurate in its projection up to the year 1990. Beyond 1990, the sharp declining trend in the actual replacement curve is very noticeable. This occurred despite a huge accumulation of bridges in the United States up to 1990. The sharp drop in actual replacements after 1990 explains the flattening in the steepness of the Kaplan-Meier survival curve at the end portion for almost every cohort, as noticed in Figure 5.

The exact reason for explaining the behavior of the actual replacement curve after 1990 is unknown. However, as mentioned in section 3.2, two possible explanations for such behavior are financial constraint which limited the number of bridges which could be replaced, and change in bridge management policy to one which is maintenance-oriented. If the former reason is the primary cause, then we should expect an increasing number of bridges which are either structurally deficient or functionally obsolete. 


\section{Evaluation of bridge performance under varying conditions}

The bridge replacement projection model derived in section 3.4 was obtained by considering bridge population of cohort 1930 of the United States as a whole, thereby in a sense assumes uniformity among all bridges in that particular cohort. However, as we know, the service life of a bridge depends heavily on the service condition (e.g. loading condition, environmental condition and etc) which the bridge is subjected to. Hence, for better representation of actual situation, it is necessary to stratify bridges in a cohort and evaluate bridge performances with respect to descriptive variables including structural aspects and dimensions, material, traffic load and geographical location.

Figure 11 gives an image of how the Kaplan-Meier survival curves for bridges will be like when (a) lumping all bridges in a cohort as a whole, and (b) stratifying the bridges in that cohort with respect to a certain variable. By performing parametric modeling individually to the stratified survival curves shown in Figure 11(b), varying projection models complying with the different classifications of a descriptive variable can be obtained, and hence the corresponding mean service lives.

Applying a diversity of bridge replacement projection models with compliance to actual service conditions is expected to raise the accuracy of projection as compared to the case where a uniform projection model is used for all bridges in a cohort (MLIT, 2002). With this expectation in mind, 
stratifications of bridge cohorts with accordance to varying conditions will be carried out in this section. Of course, it will be impossible to stratify cohorts without detailed past actual construction and replacement records with respect to each variable. Fortunately, the NBI data provided us with enough information to perform some of these stratifications. Hence, in this section, stratifications of bridge cohorts in the United States with accordance to structure length, traffic volume and truck traffic volume will be carried out.

\subsection{Stratification with accordance to structure length}

The bridges in each cohort are stratified into four structure length classifications, namely (a) $\mathrm{L}<15 \mathrm{~m}$; (b) $15 \mathrm{~m} \leq \mathrm{L}<30 \mathrm{~m}$; (c) $30 \mathrm{~m} \leq \mathrm{L}<100 \mathrm{~m}$; and (d) $\mathrm{L} \geq 100 \mathrm{~m}$. Table 2 shows the bridge population in each classification of structure length for each cohort.

The stratified Kaplan-Meier survival curves are plotted in Figure 12. From the figures, it can be observed that there is a general trend that bridges with a longer span show lower durability, especially for cohort 1930 and beyond. This trend is clearly exhibited by cohorts 1930 and 1960 . One of possible reasons is that high social importance is usually attached to long-spanned bridges and hence they get the priority of replacement. Another possible reason is that longer bridges tend to be subjected to a more severe loading condition and hence deterioration occurs faster for these bridges.

Table 3 gives a summary of the mean service life estimations when stratified by structure length, 
by applying Weibull model fitting for each stratified Kaplan-Meier survival curve. By looking across results in each row, mean service life estimations which tally well with the stratification graphs can be noted. When looking across results in each column, there is the inclination of bridge survivability decreasing with time from 1900 to 1930 , hitting the bottom line in 1930 before making an about-turn to enter an improvement phase. However, a dip in bridge performance can also be observed in cohort 1960, as noticed by low figures in mean service life for that particular cohort.

\subsection{Stratification with accordance to traffic volume}

Using information on average daily traffic (ADT) recorded for all bridges as found in the NBI database, stratification with accordance to traffic volume could be performed. However, it should be noted that the ADT coded for a bridge shows the most recent ADT count available and not the ADT at the time of replacement of the bridge should the bridge be replaced before. Due to such a constraint, an important assumption that the ADT for a bridge remains constant over time has to be made in order to rationalize any survival analysis performed with accordance to traffic volume.

Stratifications of bridge cohorts in the United States with accordance to traffic volume were then carried out. Bridge population for each traffic volume classification is summarized in Table 4 and the stratified Kaplan-Meier survival curves for each cohort are shown in Figure 13. From the graphs, although no clear trend can be observed for cohorts 1900 and 1910, for cohort 1920 and beyond, it is very obvious that bridges posed with a heavier traffic condition show a higher rate of replacement 
than bridges posed with a lighter traffic condition. This conforms with the common perception that a serious loading condition is detrimental to a structure's health.

Mean service life estimations obtained in Table 5 generally tally well with graphs shown in Figure 13. Likewise to stratification by structure length, the same transition in bridge performance over time can be noted in stratification by traffic volume. Here, again, bridge performance worsened over time from 1900 to the vicinity of 1930 , making an about-turn to enter an improvement phase after 1930 while suffering a dip in bridge performance in cohort 1960.

\subsection{Stratification with accordance to truck traffic volume}

Of special significance is the illustration of how truck traffic volume affects the survivability of a bridge, since it has been recognized widely that it is heavy vehicle such as truck, instead of passenger car, which has more impact on structural deterioration. Similar to the previous section, the assumption that the average daily truck traffic for a bridge remains constant over time will also be made here.

Table 6 shows the bridge populations for the different truck traffic volume classifications in each cohort. Notice that bridges constructed in the early period (i.e. 1900, 1910 and 1920) are subjected to a relatively lighter truck traffic load; perhaps the result of the substandard conditions of these bridges due to aging which limited their load carrying capacities.

From Figure 14, while no clear trend can be observed for cohorts 1900 and 1910, distinct 
dispersion in Kaplan-Meier survival curves can be seen in the post-1920 cohorts, implying that bridges subjected to a heavier truck traffic condition are at greater mortality risk than those subjected to a lighter truck traffic condition. As compared with the earlier two cases i.e. stratifications with accordance to structure length and traffic volume, it can be noted that truck traffic volume clearly has more effect on the mortality of bridges, as observed from the large divergence in survival curves between each truck traffic volume classification.

From the mean service life estimations shown in Table 7, again, it can be observed that cohorts 1930 and 1960 are the cohorts with the worst performances. Notice that so far, in all three types of stratifications (i.e. structure length, traffic volume and truck traffic volume), either cohort 1930 and its vicinity or cohort 1960 is the cohort which shows the lowest durability. Since, as we had noted from Figure 9 that construction booms in the United States occurred in the vicinities of 1930 and 1960, this has implications that bridges constructed during these periods of construction boom are of inferior quality when compared to bridges constructed in other times. In fact, the same phenomenon too is known to occur in Japan, since it is well-known that bridges built during the high economic growth period of the 1960s and 1970s generally deteriorate faster due to poor quality control caused by mass construction and hastened work. Hence, the same problem can be considered possible in the United States. 


\subsection{Expectations of adopting a more microscopic approach}

Table 8 gives a summary of the bridge replacement projection models for the representative cohort (cohort 1930) of the United States. Using the maximum likelihood estimates for the unknown parameters $\alpha$ and $\lambda$ of the Weibull distribution as shown in the table, the probability function can be plotted for each classification by applying the formula given in equation (12). Each probability function will then serve as the bridge replacement projection model for that classification of bridges.

By adopting such a relatively microscopic approach towards projecting future bridge replacement demand, the accuracy of projection is expected to be improved, as compared to using one uniform projection model for all bridges in a cohort. For instance, instead of treating all bridges as a whole and projecting by using the uniform model obtained in section 3.4, grouping the bridges according to the four given classifications for traffic volume shown in Table 8, before projecting by using the four distinct projection models obtained is more likely to raise the accuracy level of the projection results.

\section{Conclusions}

Using the method of survival analysis on bridge survival data of Japan and the United States, a bridge replacement projection model was successfully derived. In the process leading up to the derivation of the projection model, two major findings are listed as follows:

- Bridge performance between Japan and the United States is very much alike, at least in the early 
stage, despite all the differences in social and geographical conditions between the two countries.

- The mean service life of a bridge is estimated at 93.5 years, which is significantly longer than the depreciation service life set at 60 years.

The validity of the derived bridge replacement projection model, which was addressed as the proposed projection model, was verified when it was found out that the projection results generated by it were relatively closer to actual replacements in the United States for the past, as compared to projection results obtained by using conventional projection methods.

Based on the findings and the validation, it can be claimed that the bridge replacement projection model obtained for the United States is applicable as an approximation for other countries where either the Japanese or the American specifications are followed in the design of their bridges.

By carrying out stratifications of bridge cohorts in the United States, bridges with a longer structure length, heavier traffic condition or heavier truck traffic condition were shown to have shorter lifespans according to the stratification results. Also, it was noted that bridges constructed during periods of construction boom were of inferior quality when compared to those constructed in other times.

As a result of stratification, different projection models (by structure length; traffic volume; or truck traffic volume) conforming to any classification of bridge were obtained. Through the application of a diversity of projection models with compliance to actual service conditions, the 
accuracy of projection is expected to be further improved, since a more microscopic approach had been adopted.

It is believed that the projection results obtained by using these projection models will provide a good insight into future scene on bridge replacement needs; which in turn is hoped to be utilized as a useful tool in budget allocation decision-making process.

\section{Acknowledgements}

Survival analysis on bridges will be impossible to carry out without detailed past bridge data provided by PWRI of Japan and FHWA. The authors are therefore thankful for the opportunity to use these data in this study.

\section{References}

ASCE, Report Card for America's Infrastructure, 2003 Progress Report, 2003 (American Society of Civil Engineers)

Collett, D., Modelling survival data in medical research, 1994 (Chapman \& Hall)

FHWA, National Bridge Inventory (NBI), 2003a, (Federal Highway Administration: U.S. Department of Transportation) 
FHWA, Recording and coding guide for the structure inventory and appraisal of the nation's bridges, Report No. FHWA-PD-96-001, 2003b, (Federal Highway Administration: U.S. Department of Transportation).

JHUC, Doro tokei nenpo, 1964 2003, Japan Highway Users Conference (Ministry of Land, Infrastructure and Transport: Japan). (In Japanese)

Kalbfleisch, J. D., and Prentice, R. L., The statistical analysis of failure time data, 1980 (John Wiley \& Sons, Inc.).

Klein, J. P., and Moeschberger, M. L., Survival analysis: Techniques for censored and truncated data, 1997 (Springer).

Lawless, J. F., Statistical models and methods for lifetime data, 2003 (John Wiley \& Sons, Inc.).

MOF, Genka syoukyaku sisan no taiyou nensu nado ni kan suru syourei (26th revision), 1998

(Ministry of Finance: Japan). (In Japanese)

MLIT, Kongo no shakai sihon seibi ni tsuite no kiso teki kenkyu, Policy Research Institute for Land, Infrastructure and Transport, 2002 (Ministry of Land, Infrastructure and Transport: Japan). (In Japanese)

Newman, S. C., Biostatistical methods in epidemiology, 2001 (John Wiley \& Sons, Inc.).

OECD, Bridge management, 1992, Road Transport Research (Organization for Economic Co-operation and Development).

PWRI., Kyoryo no kakekae ni kan suru chosa kekka ( I, II and III), 1977, 1986, 1996, Public Works Research Institute (Ministry of Construction: Japan). (In Japanese) 
Table 1. Depreciation service lives of infrastructure

\begin{tabular}{l|c||l|c}
\hline Type of infrastructure & Service life & Type of infrastructure & Service life \\
\hline \hline Road (with concrete pavement) & 15 & Sediment control dam (concrete) & 30 \\
Road (with asphalt pavement) & 10 & Retarding basin & 60 \\
Bridge (RC) & 60 & Seawall (concrete) & 30 \\
Tunnel (concrete) & 30 & Water gate & 45 \\
River wall (concrete) & 30 & Rockfill dam & 50 \\
\hline
\end{tabular}


Table 2. Bridge population in each cohort, stratified by structure length

\begin{tabular}{|c|c|c|c|c|c|}
\hline Cohort & $\mathrm{L}<15 \mathrm{~m}$ & $15 \mathrm{~m} \leq \mathrm{L}<30 \mathrm{~m}$ & $30 \mathrm{~m} \leq \mathrm{L}<100 \mathrm{~m}$ & $\mathrm{~L} \geq 100 \mathrm{~m}$ & Total \\
\hline \hline 1900 & 5,126 & 2,051 & 1,066 & 89 & 8,332 \\
\hline 1910 & 1,053 & 826 & 474 & 48 & 2,401 \\
\hline 1920 & 3,222 & 1,100 & 495 & 62 & 4,879 \\
\hline 1930 & 7,622 & 2,446 & 1,440 & 326 & 11,834 \\
\hline 1940 & 8,015 & 2,306 & 1,455 & 286 & 12,062 \\
\hline 1950 & 7,685 & 2,582 & 2,295 & 487 & 13,049 \\
\hline 1960 & 7,935 & 3,389 & 6,591 & 1,244 & 19,159 \\
\hline 1970 & 5,851 & 2,825 & 6,597 & 1,719 & 16,992 \\
\hline
\end{tabular}


Table 3. Mean service life estimations when stratified by structure length

\begin{tabular}{|c|c|c|c|c|}
\hline Sohort & $\mathrm{L}<15 \mathrm{~m}$ & $15 \mathrm{~m} \leq \mathrm{L}<30 \mathrm{~m}$ & $30 \mathrm{~m} \leq \mathrm{L}<100 \mathrm{~m}$ & $\mathrm{~L} \geq 100 \mathrm{~m}$ \\
\hline \hline 1900 & 111.1 & 108.2 & 112.4 & 116.3 \\
\hline 1910 & 113.1 & 115.2 & 118.4 & 120.3 \\
\hline 1920 & 114.3 & 106.0 & 118.8 & 118.1 \\
\hline 1930 & 116.2 & 105.1 & 95.6 & 76.0 \\
\hline 1940 & 115.8 & 108.4 & 101.0 & 81.1 \\
\hline 1950 & 117.8 & 110.3 & 111.6 & 98.0 \\
\hline 1960 & 117.3 & 91.1 & 77.7 & 76.3 \\
\hline 1970 & 144.0 & 114.1 & 106.9 & 126.6 \\
\hline
\end{tabular}


Table 4. Bridge population in each cohort, stratified by traffic volume

\begin{tabular}{|c|c|c|c|c|c|}
\hline Cohort & $\leq 1,000$ & $1,001 \sim 5,000$ & $5,001 \sim 10,000$ & $\geq 10,001$ & Total \\
\hline \hline 1900 & 6,793 & 753 & 289 & 497 & 8,332 \\
\hline 1910 & 2,037 & 190 & 71 & 103 & 2,401 \\
\hline 1920 & 4,070 & 477 & 190 & 142 & 4,879 \\
\hline 1930 & 8,134 & 2,192 & 748 & 760 & 11,834 \\
\hline 1940 & 8,653 & 2,101 & 601 & 707 & 12,062 \\
\hline 1950 & 8,631 & 2,263 & 747 & 1,408 & 13,049 \\
\hline 1960 & 9,801 & 3,125 & 1,534 & 4,699 & 19,159 \\
\hline
\end{tabular}


Table 5. Mean service life estimations when stratified by traffic volume

\begin{tabular}{|c|c|c|c|c|}
\hline Cohort & $\leq 1,000$ & $1,001 \sim 5,000$ & $5,001 \sim 10,000$ & $\geq 10,001$ \\
\hline \hline 1900 & 108.3 & 117.0 & 121.3 & 154.5 \\
\hline 1910 & 114.5 & 111.9 & 106.6 & 126.3 \\
\hline 1920 & 112.4 & 97.8 & 92.5 & 94.0 \\
\hline 1930 & 119.0 & 94.3 & 84.5 & 81.6 \\
\hline 1940 & 115.3 & 105.7 & 85.1 & 86.1 \\
\hline 1950 & 117.0 & 119.7 & 94.7 & 89.4 \\
\hline 1970 & 108.3 & 100.2 & 81.2 & 69.5 \\
\hline
\end{tabular}


Table 6. Bridge population in each cohort, stratified by truck traffic volume

\begin{tabular}{|c|c|c|c|c|c|}
\hline Cohort & $\leq 100$ & $101 \sim 500$ & $501 \sim 1,000$ & $\geq 1,001$ & Total \\
\hline \hline 1900 & 7,422 & 552 & 165 & 193 & 8,332 \\
\hline 1910 & 2,188 & 133 & 47 & 33 & 2,401 \\
\hline 1920 & 4,320 & 374 & 128 & 57 & 4,879 \\
\hline 1930 & 8,956 & 1,821 & 590 & 467 & 11,834 \\
\hline 1940 & 9,378 & 1,701 & 543 & 440 & 12,062 \\
\hline 1950 & 9,900 & 1,784 & 555 & 810 & 13,049 \\
\hline 1960 & 11,740 & 2,581 & 1,228 & 3,610 & 19,159 \\
\hline 1970 & 10,228 & 2,105 & 1,168 & 3,491 & 16,992 \\
\hline
\end{tabular}


Table 7. Mean service life estimations when stratified by truck traffic volume

\begin{tabular}{|c|c|c|c|c|}
\hline Cohort & $\leq 100$ & $101 \sim 500$ & $501 \sim 1,000$ & $\geq 1,001$ \\
\hline \hline 1900 & 109.8 & 108.2 & 122.3 & 129.0 \\
\hline 1910 & 115.1 & 112.2 & 100.8 & 95.1 \\
\hline 1920 & 112.8 & 94.4 & 74.2 & 73.8 \\
\hline 1930 & 117.4 & 90.0 & 72.2 & 60.1 \\
\hline 1940 & 118.1 & 103.2 & 81.3 & 72.9 \\
\hline 1950 & 117.0 & 119.7 & 95.1 & 66.8 \\
\hline 1960 & 112.3 & 99.2 & 72.4 & 63.3 \\
\hline 1970 & 117.7 & 144.2 & 111.5 & 100.9 \\
\hline
\end{tabular}


Table 8. Summary of projection models for the representative bridge cohort of the United States

\begin{tabular}{|c|c|c|c|c|c|}
\hline \multirow{2}{*}{ Stratification by } & \multirow{2}{*}{ Classification } & \multirow{2}{*}{ Number of Bridges } & \multicolumn{2}{|c|}{ Parameter Estimates } & \multirow{2}{*}{$\begin{array}{c}\text { Mean Service Life } \\
\text { (Years) }\end{array}$} \\
\hline & & & $\alpha$ & $\lambda$ & \\
\hline \multirow{4}{*}{ Structure Length } & $<15 \mathrm{~m}$ & 7,622 & 1.842 & $7.645 \times 10^{-3}$ & 116.2 \\
\hline & $15 \mathrm{~m} \sim 30 \mathrm{~m}$ & 2,446 & 1.927 & $8.436 \times 10^{-3}$ & 105.1 \\
\hline & $30 \mathrm{~m} \sim 100 \mathrm{~m}$ & 1,440 & 1.931 & $9.277 \times 10^{-3}$ & 95.6 \\
\hline & $\geq 100 \mathrm{~m}$ & 326 & 2.364 & $11.662 \times 10^{-3}$ & 76.0 \\
\hline \multirow{4}{*}{ Traffic Volume } & $\leq 1,000$ & 8,134 & 1.976 & $7.449 \times 10^{-3}$ & 119.0 \\
\hline & $1,001 \sim 5,000$ & 2,192 & 1.770 & $9.437 \times 10^{-3}$ & 94.3 \\
\hline & $5,001 \sim 10,000$ & 748 & 1.855 & $10.509 \times 10^{-3}$ & 84.5 \\
\hline & $\geq 10,001$ & 760 & 1.923 & $10.871 \times 10^{-3}$ & 81.6 \\
\hline \multirow{4}{*}{$\begin{array}{c}\text { Truck Traffic } \\
\text { Volume }\end{array}$} & $\leq 100$ & 8,956 & 2.079 & $7.548 \times 10^{-3}$ & 117.4 \\
\hline & $101 \sim 500$ & 1,821 & 1.642 & $9.936 \times 10^{-3}$ & 90.0 \\
\hline & $501 \sim 1,000$ & 590 & 1.887 & $12.288 \times 10^{-3}$ & 72.2 \\
\hline & $\geq 1,001$ & 467 & 2.016 & $14.739 \times 10^{-3}$ & 60.1 \\
\hline
\end{tabular}




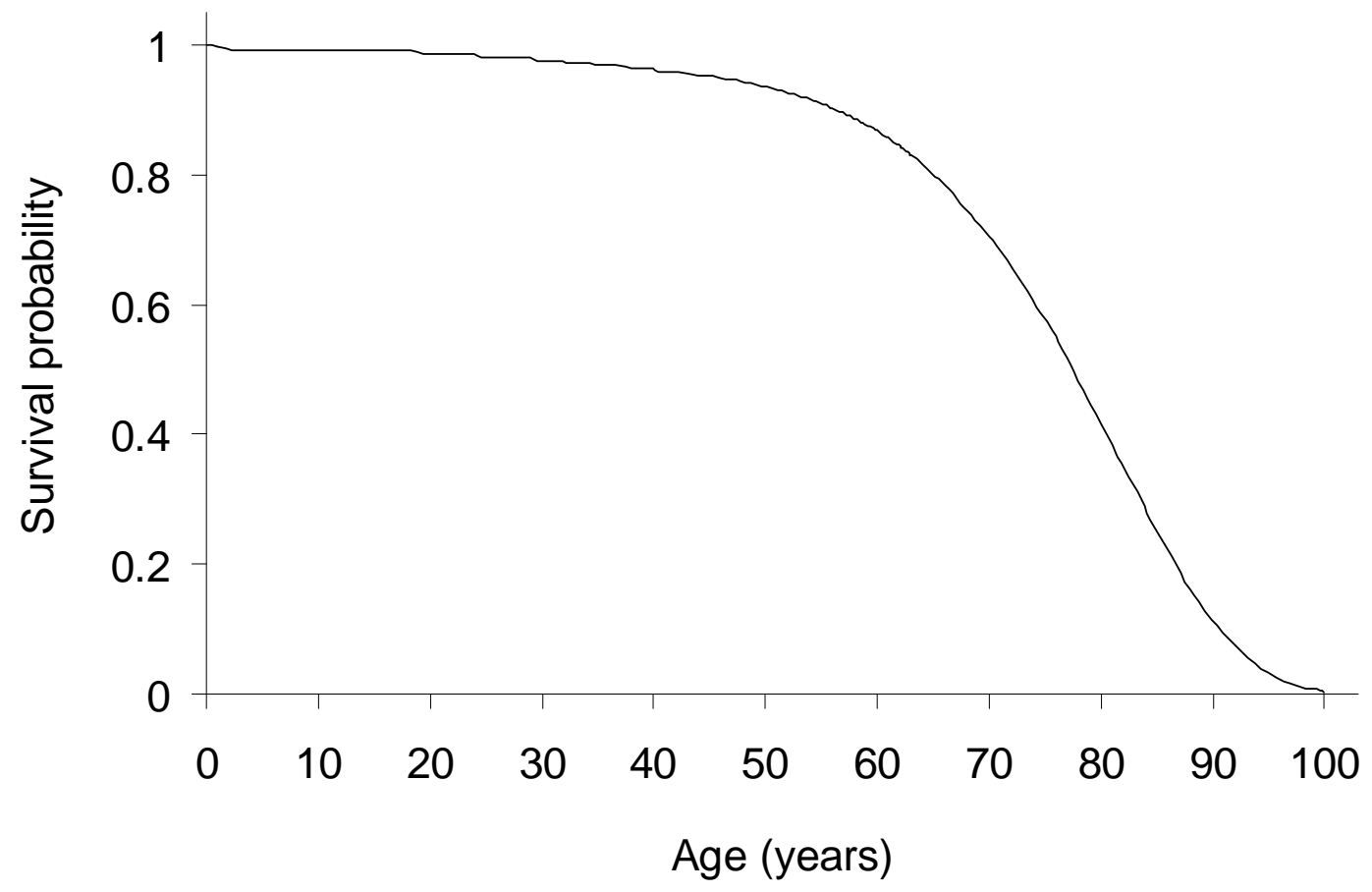

(a) 


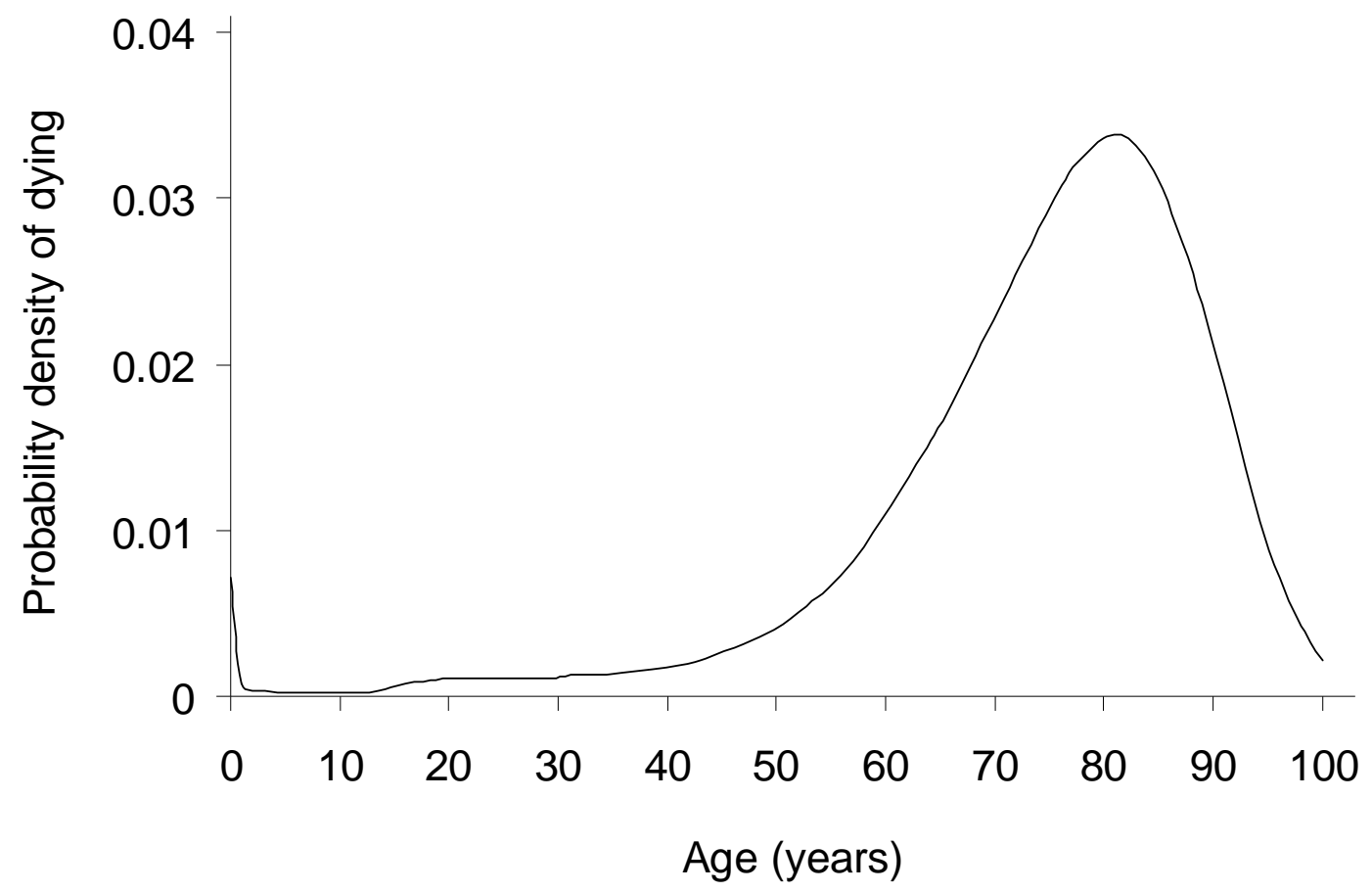

(b) 


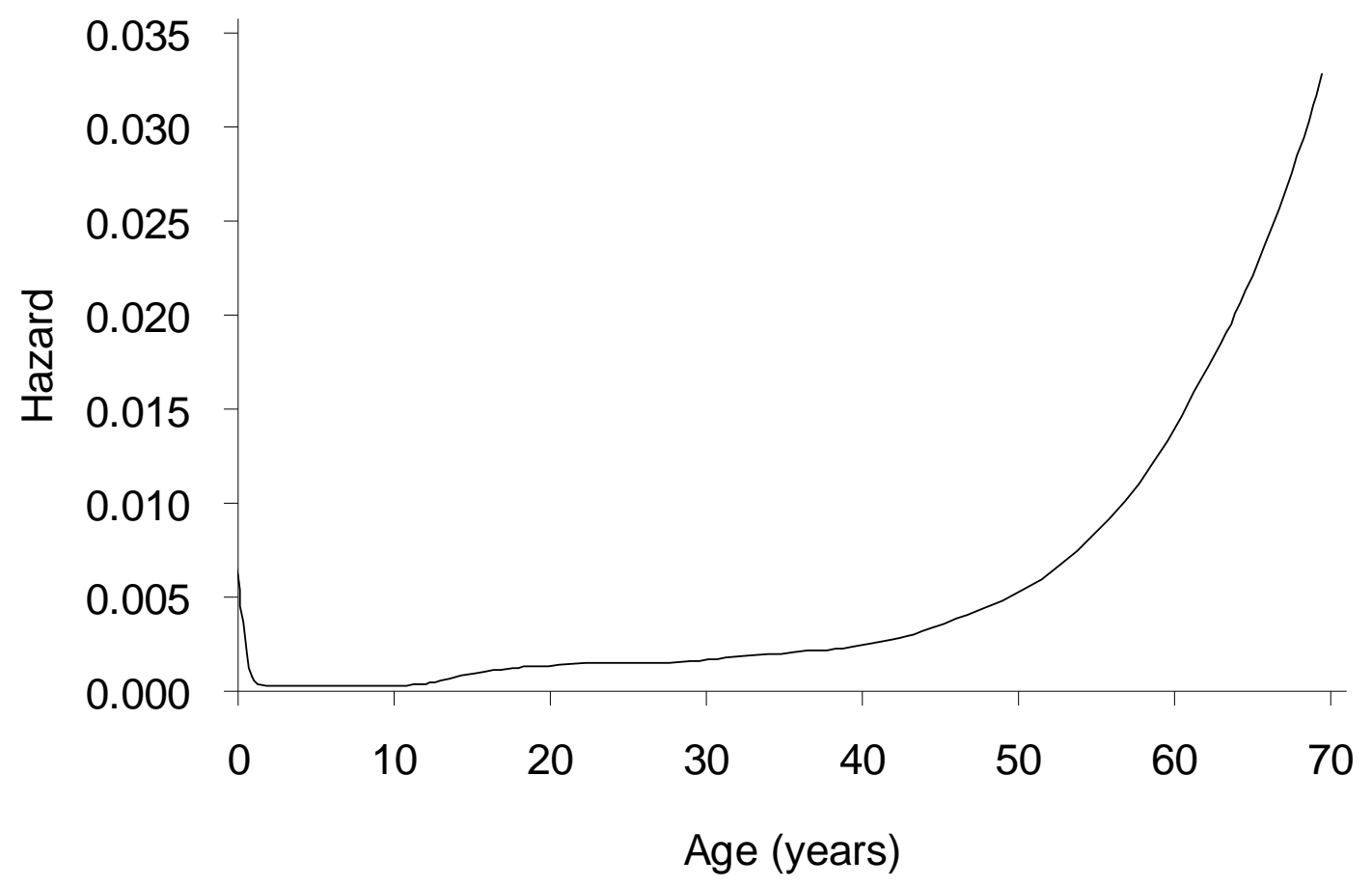

(c)

Figure 1. (a) Survival function, (b) Probability function, (c) Hazard function, for a hypothetical human cohort 


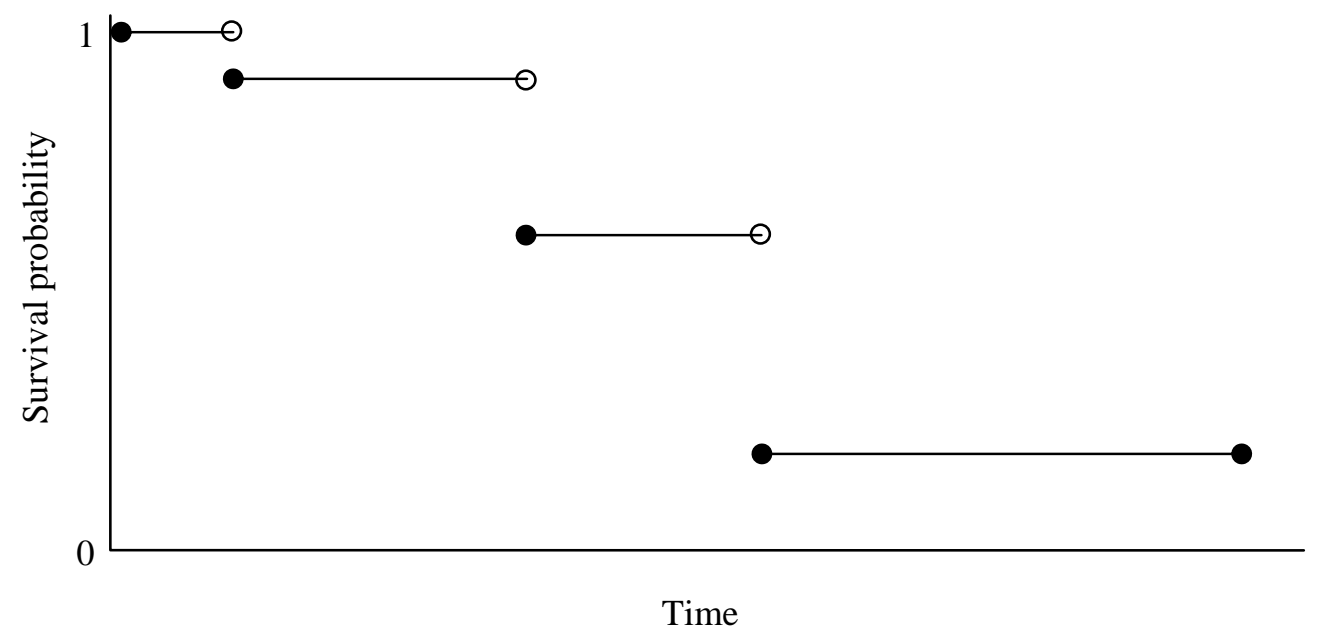

Figure 2. Schematic Kaplan-Meier survival curve 


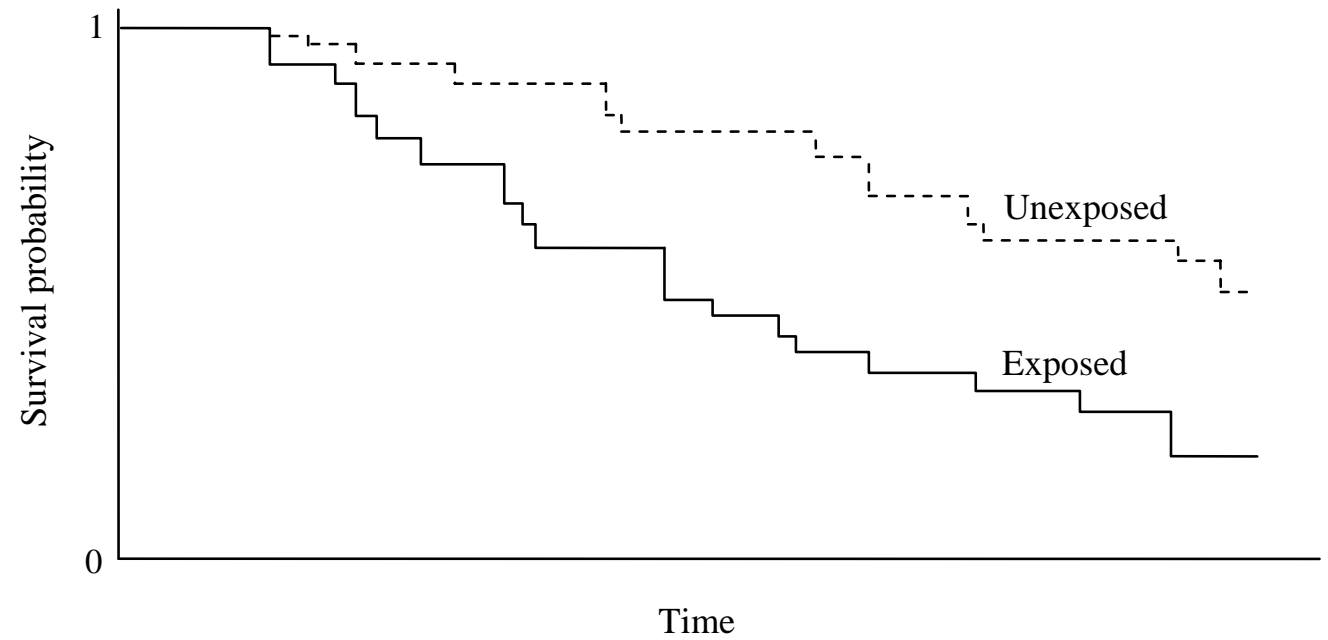

Figure 3. Kaplan-Meier survival curves: Stratification by exposure 


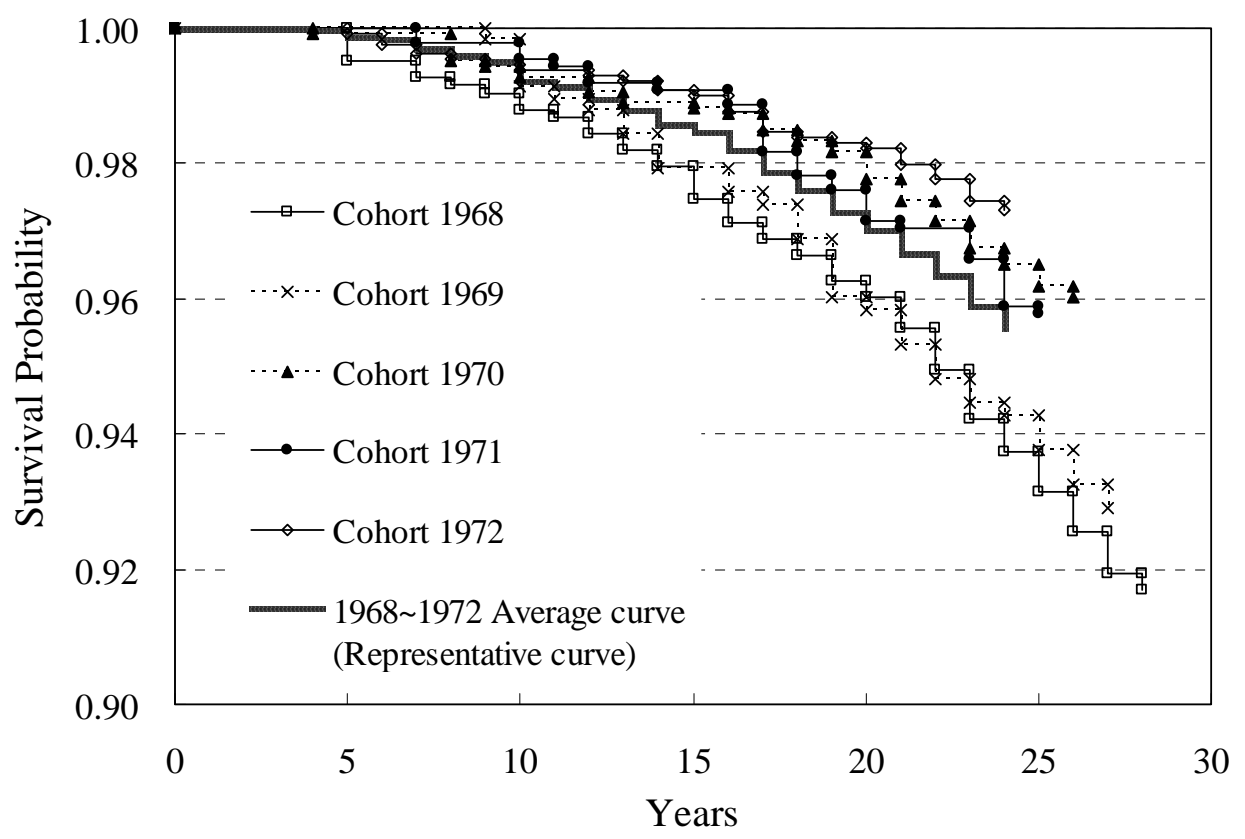

Figure 4. Kaplan-Meier survival curves for bridges in Japan 


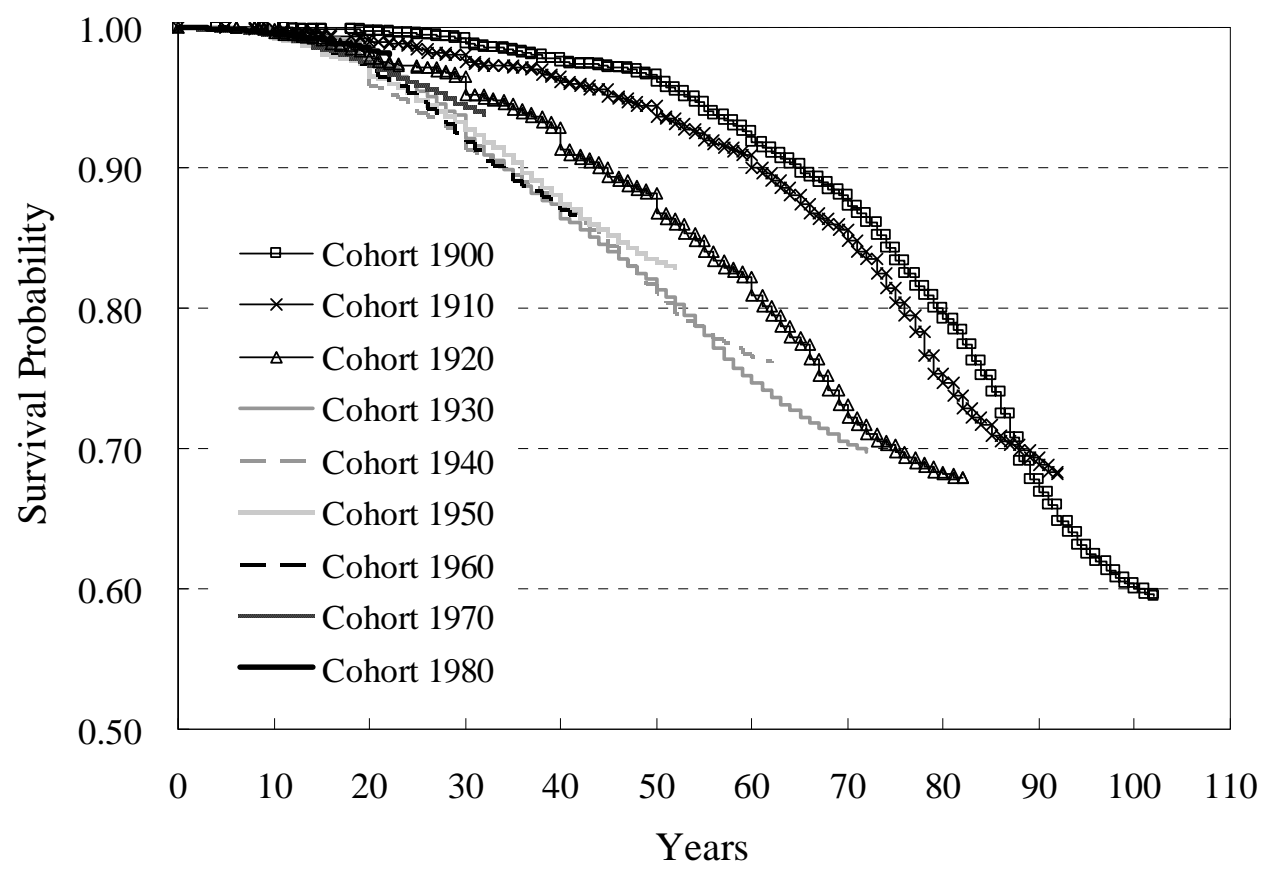

Figure 5. Kaplan-Meier survival curves for bridges in the United States 


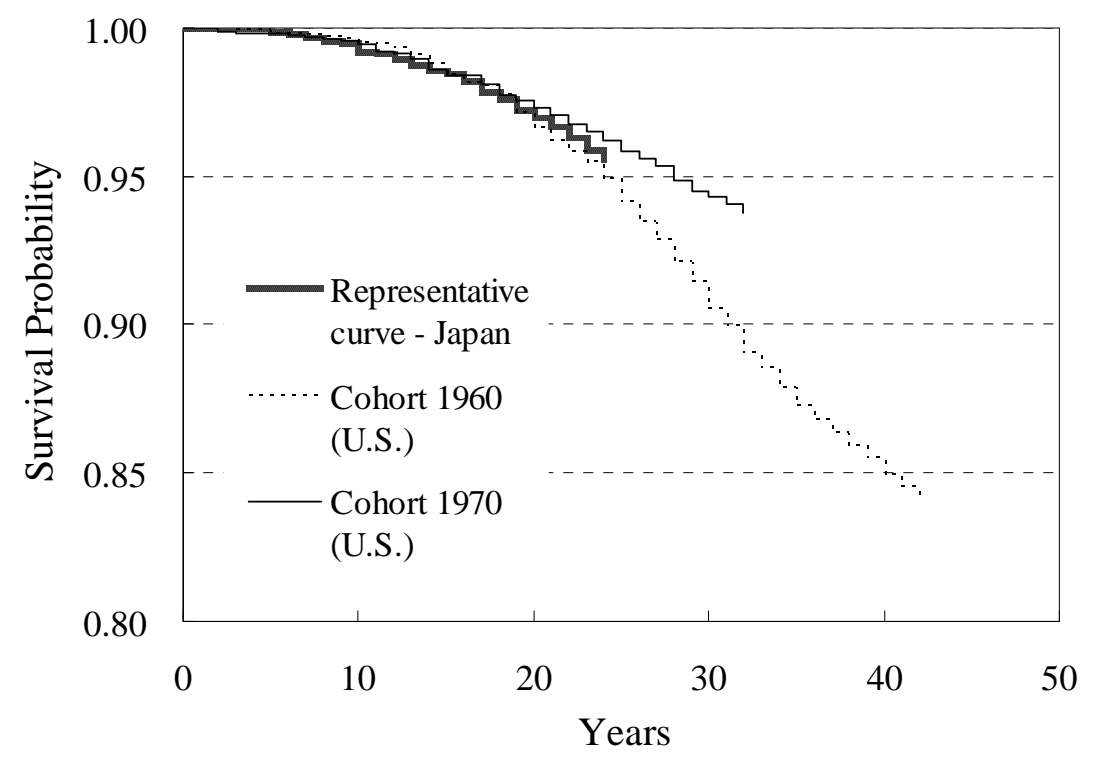

Figure 6. Comparison of Kaplan-Meier survival curves for bridges in Japan and the United States 


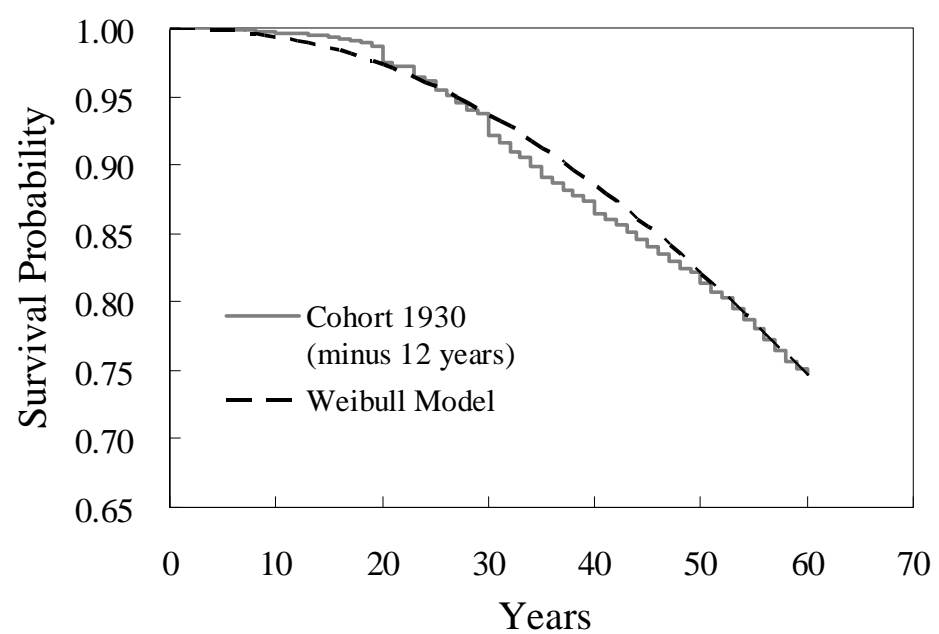

Figure 7. Weibull and Kaplan-Meier survival curves for the representative bridge cohort of the United States 


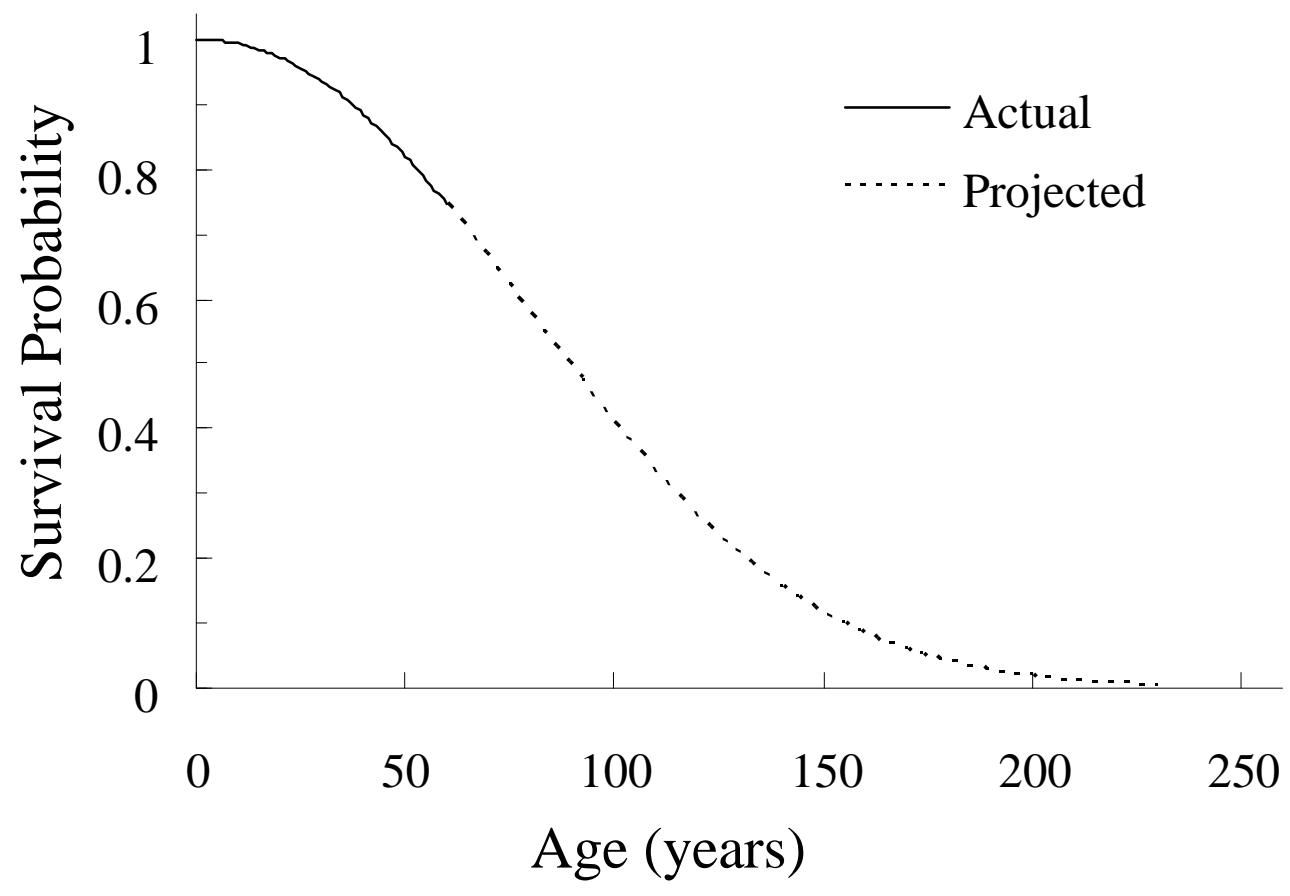

(a) 


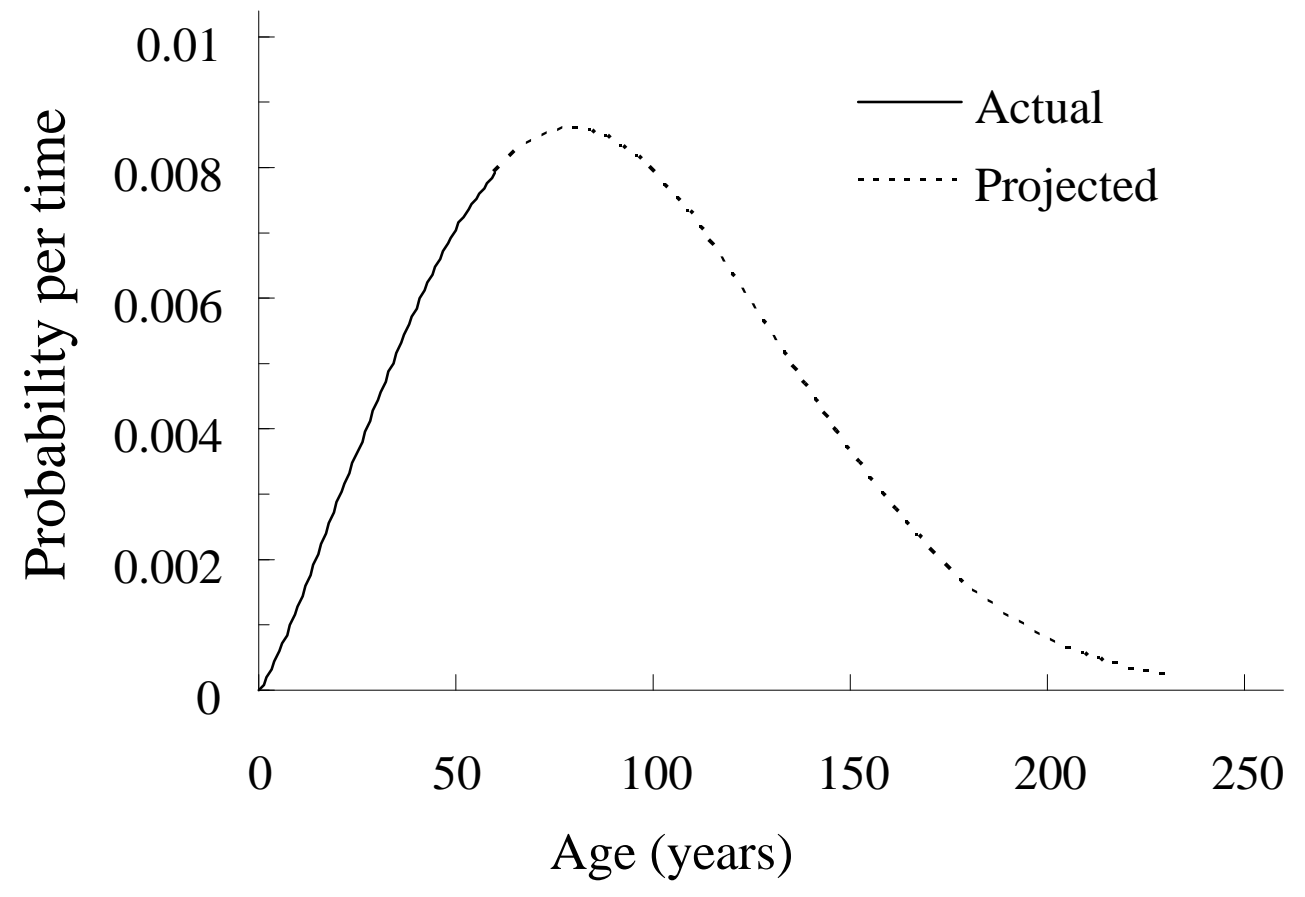

(b) 


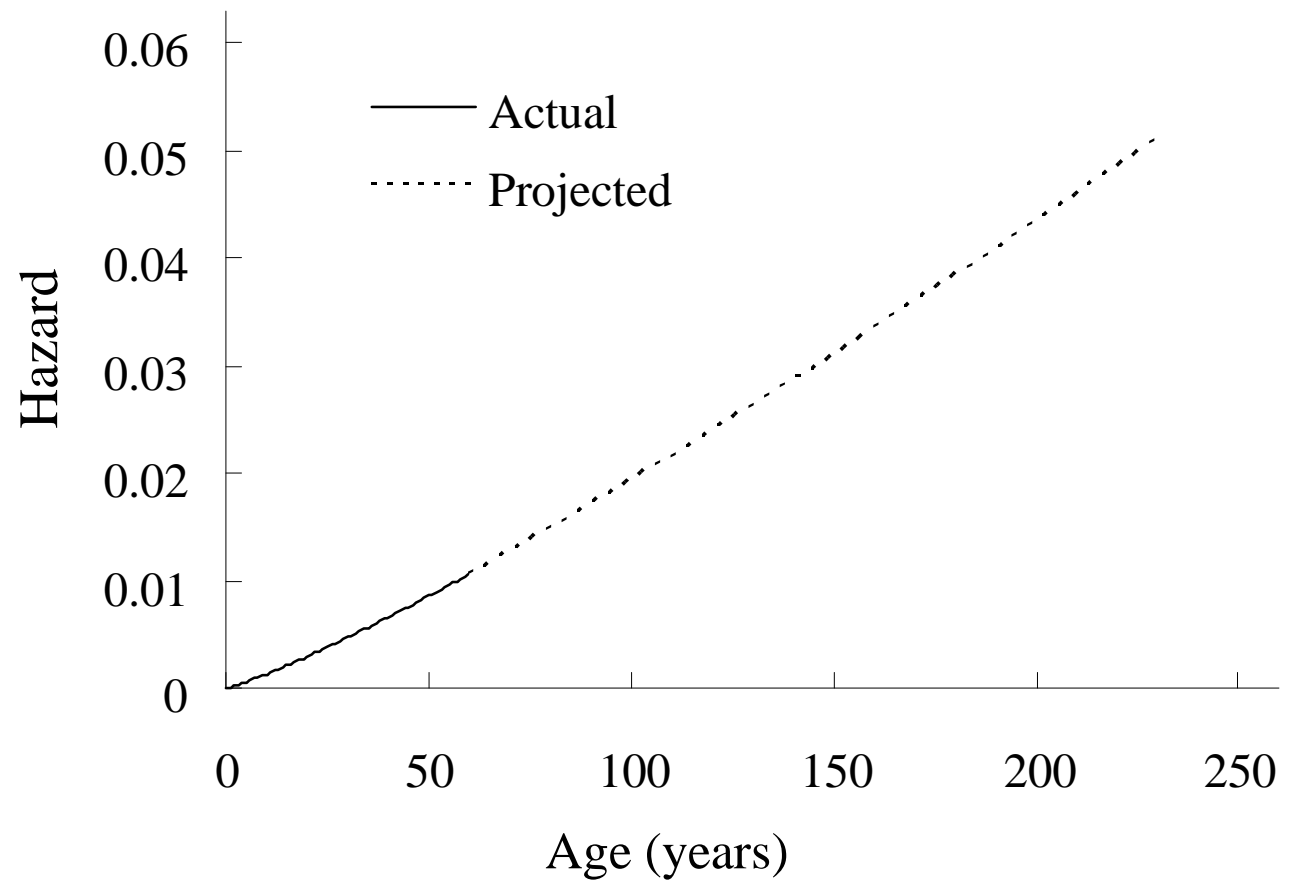

(c)

Figure 8.(a) Survival function, (b) Probability function, (c) Hazard function, for the representative bridge cohort of the United States 


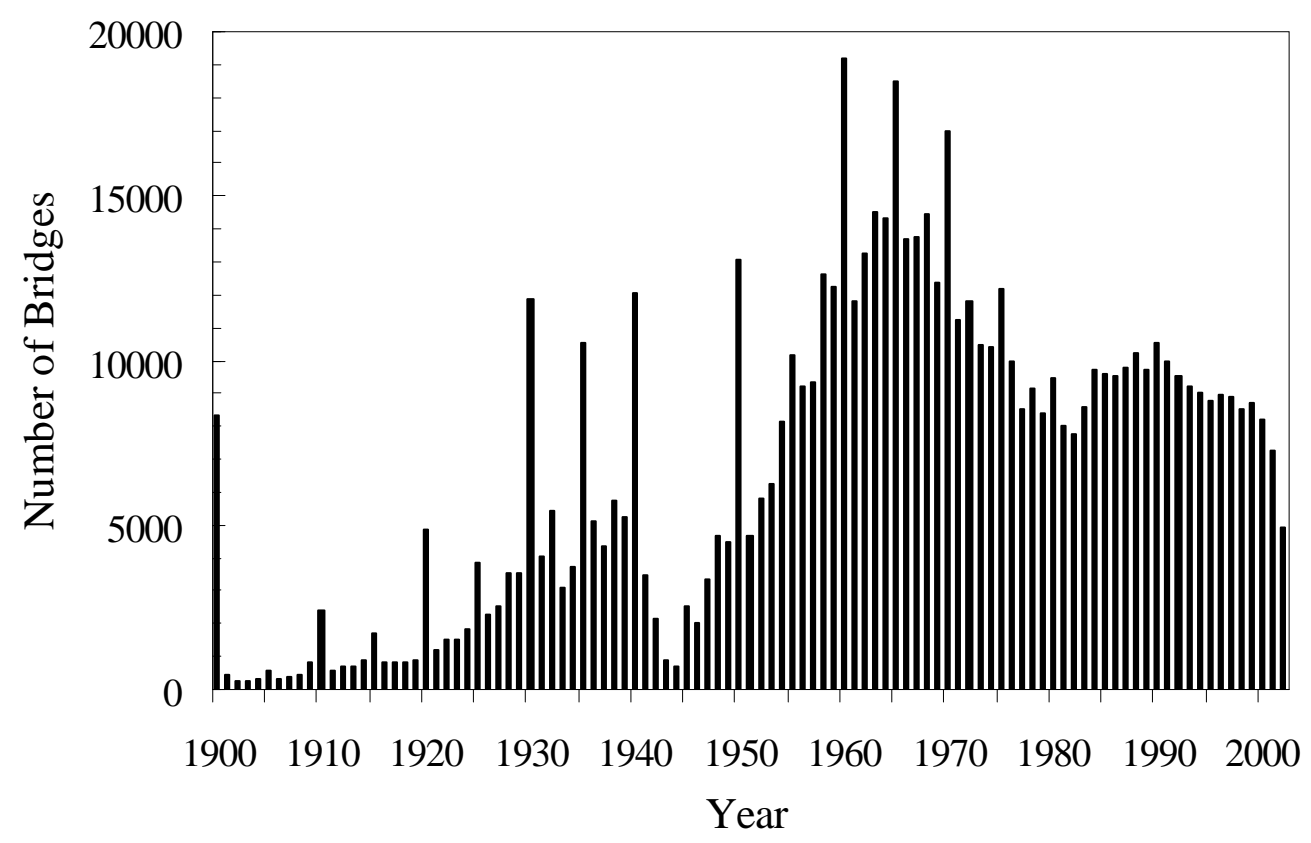

Figure 9. Number of bridges built yearly in the United States, 1900 2002 


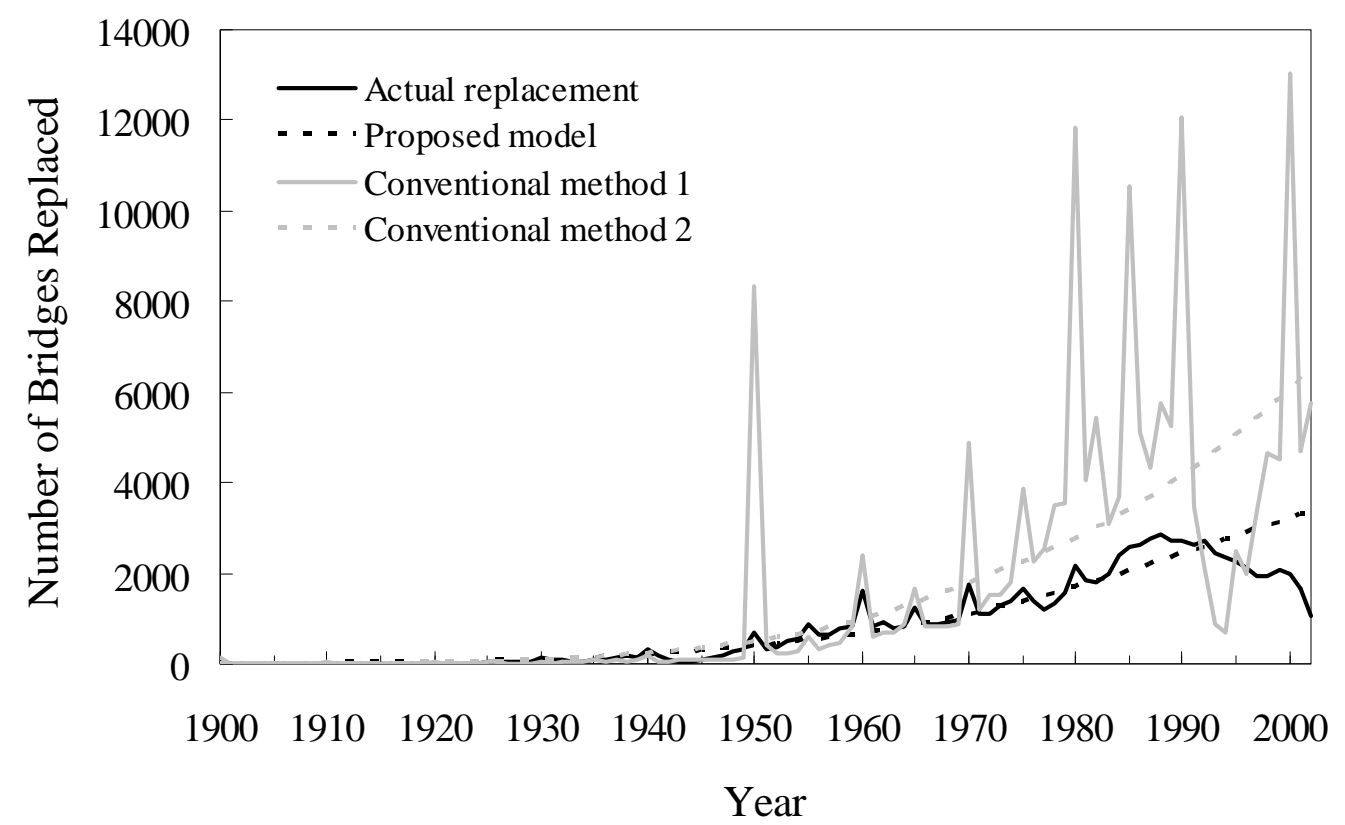

Figure 10. Actual and projected replacements of bridges in the United States, 1900 2002 


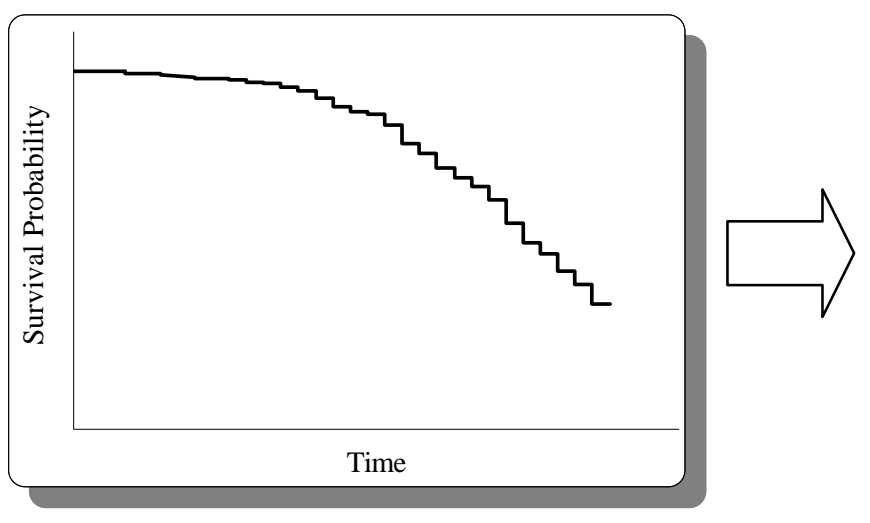

(a)

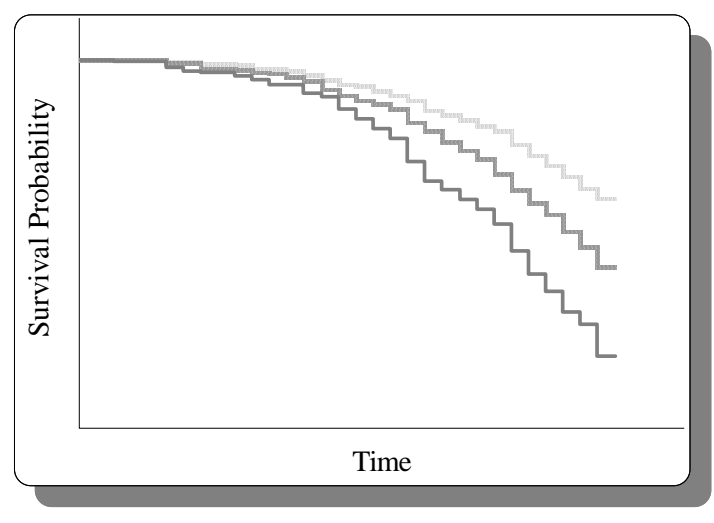

(b)

Figure 11. Kaplan-Meier survival curves for bridges in a cohort (a) Without stratification (b) With stratification by a certain variable 


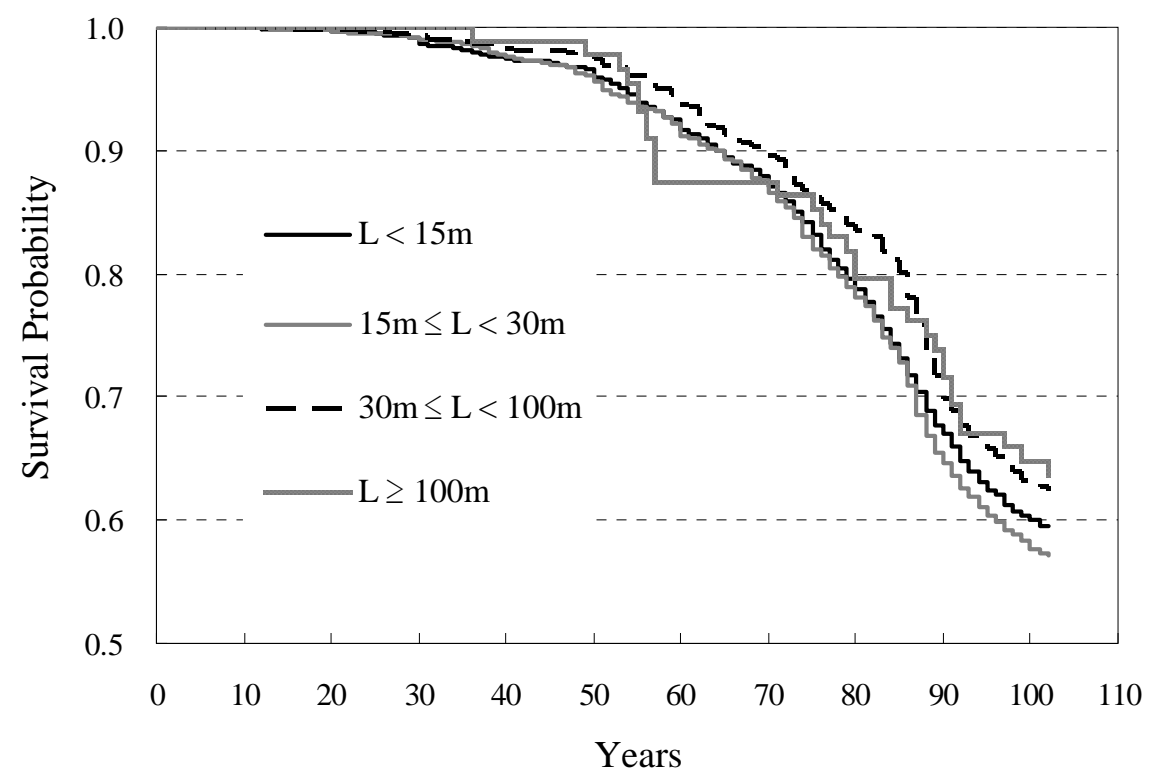

(a) Cohort 1900 


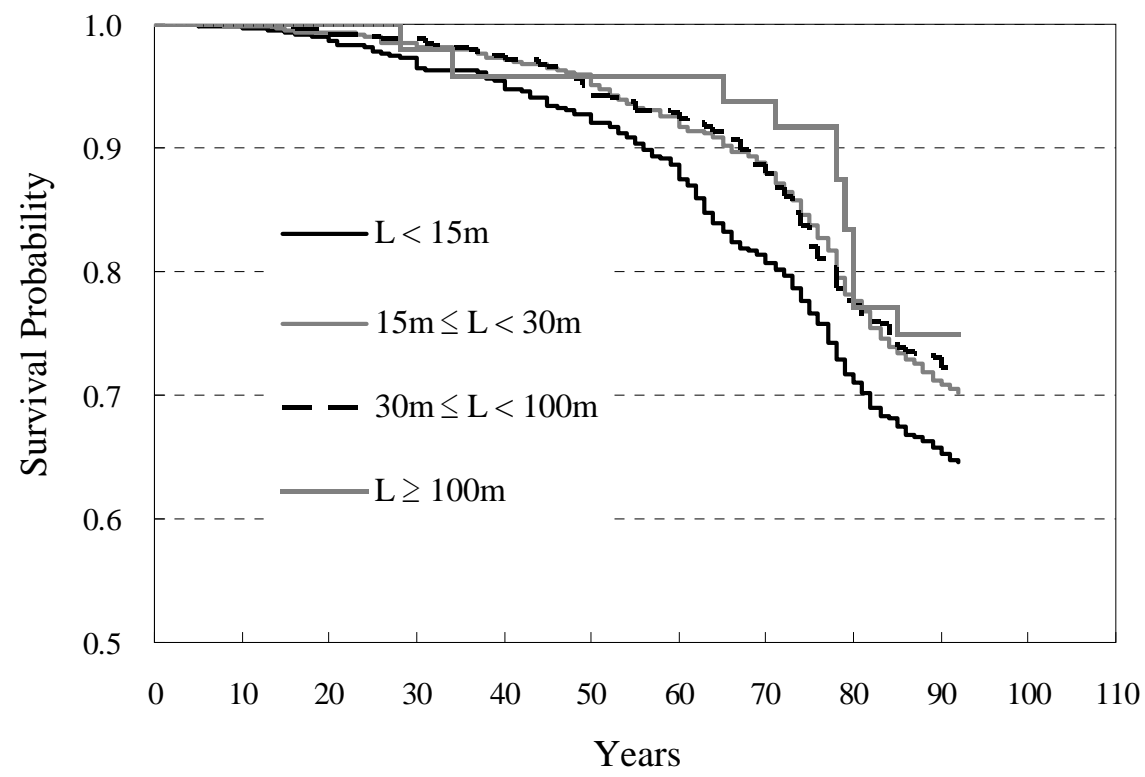

(b) Cohort 1910 


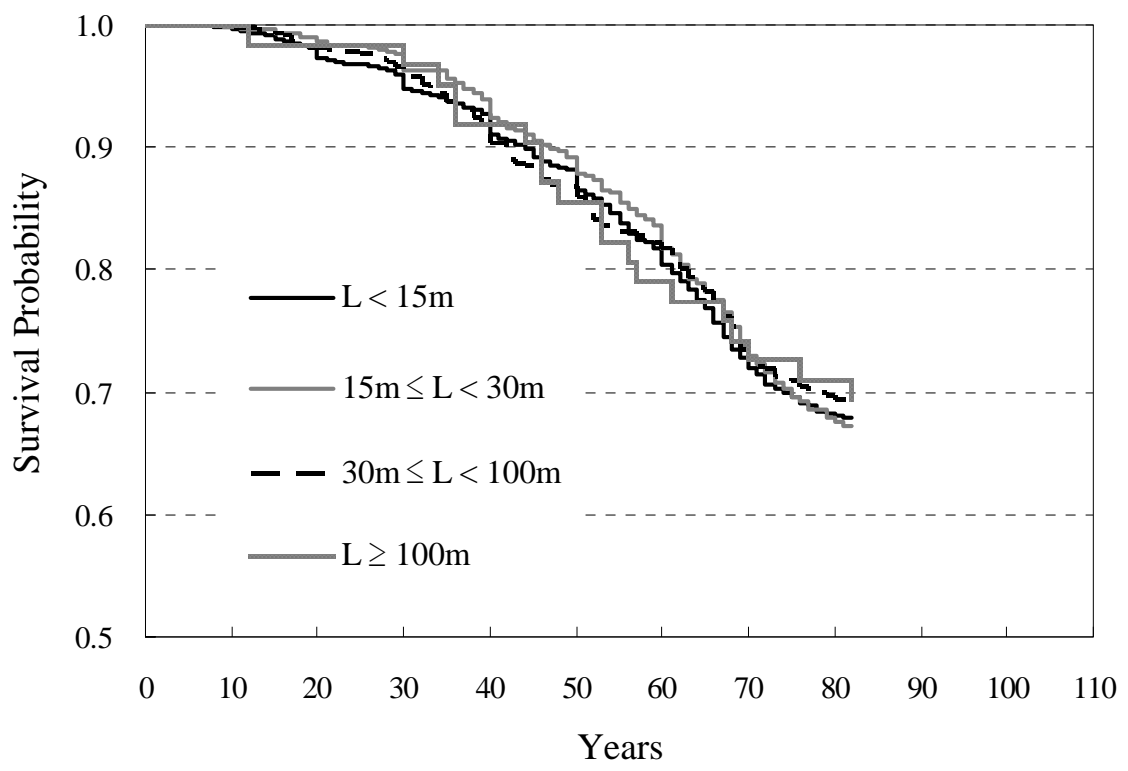

(c) Cohort 1920 


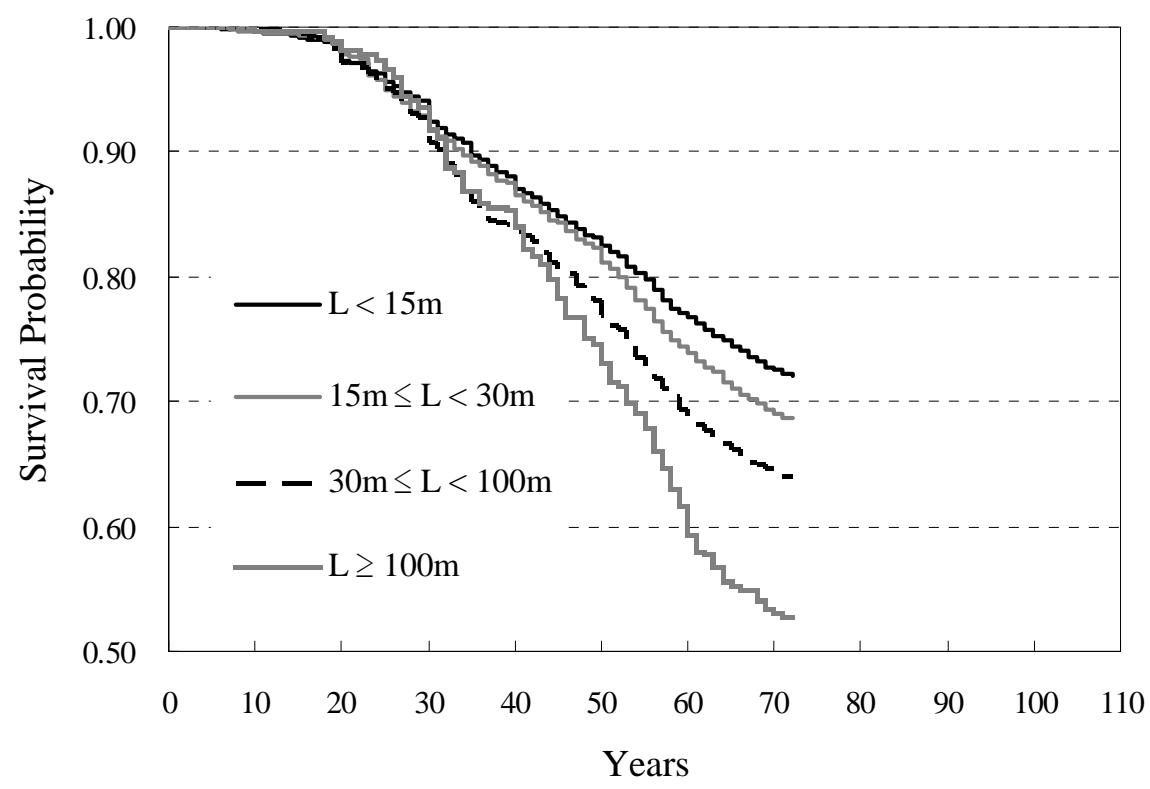

(d) Cohort 1930 


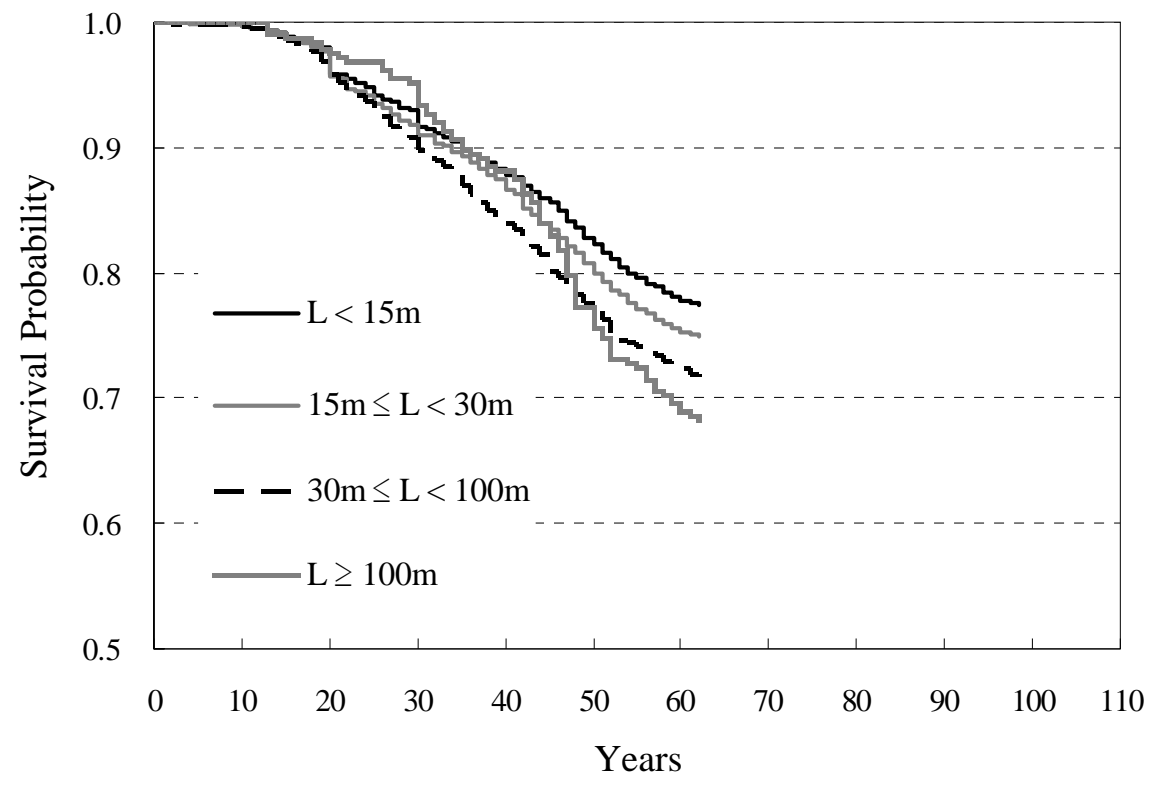

(e) Cohort 1940 


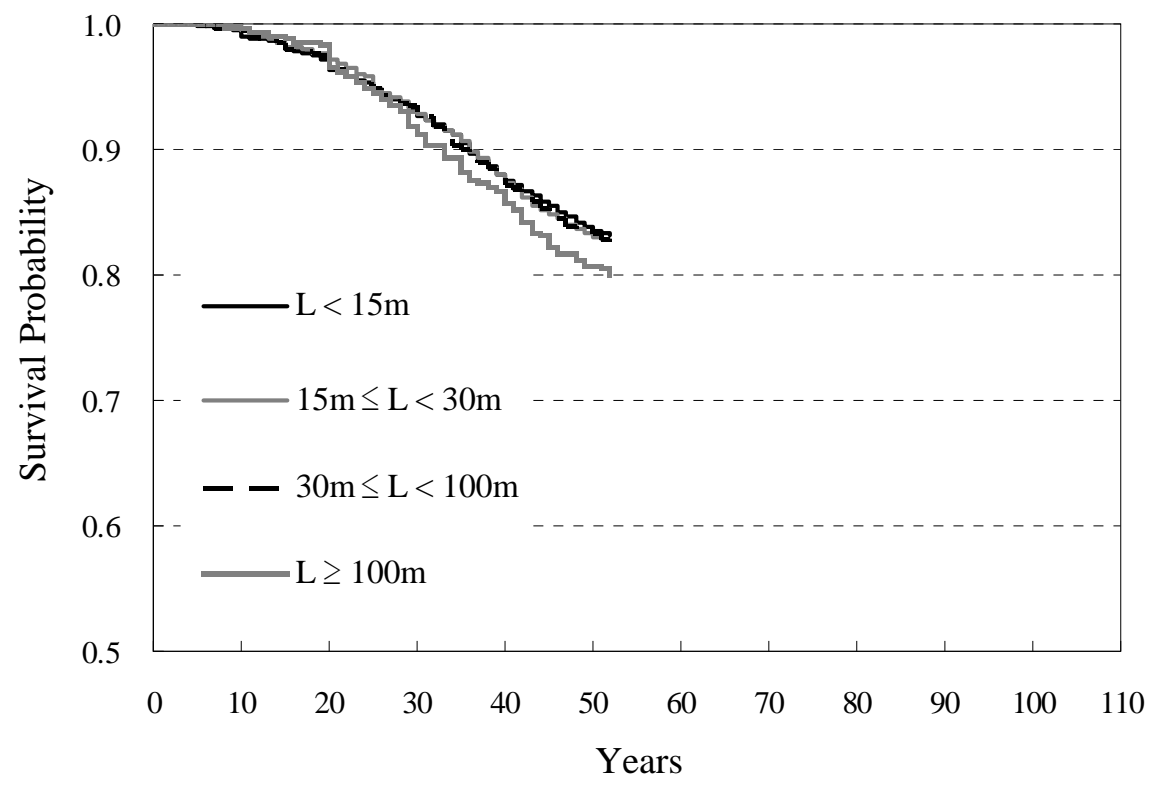

(f) Cohort 1950 


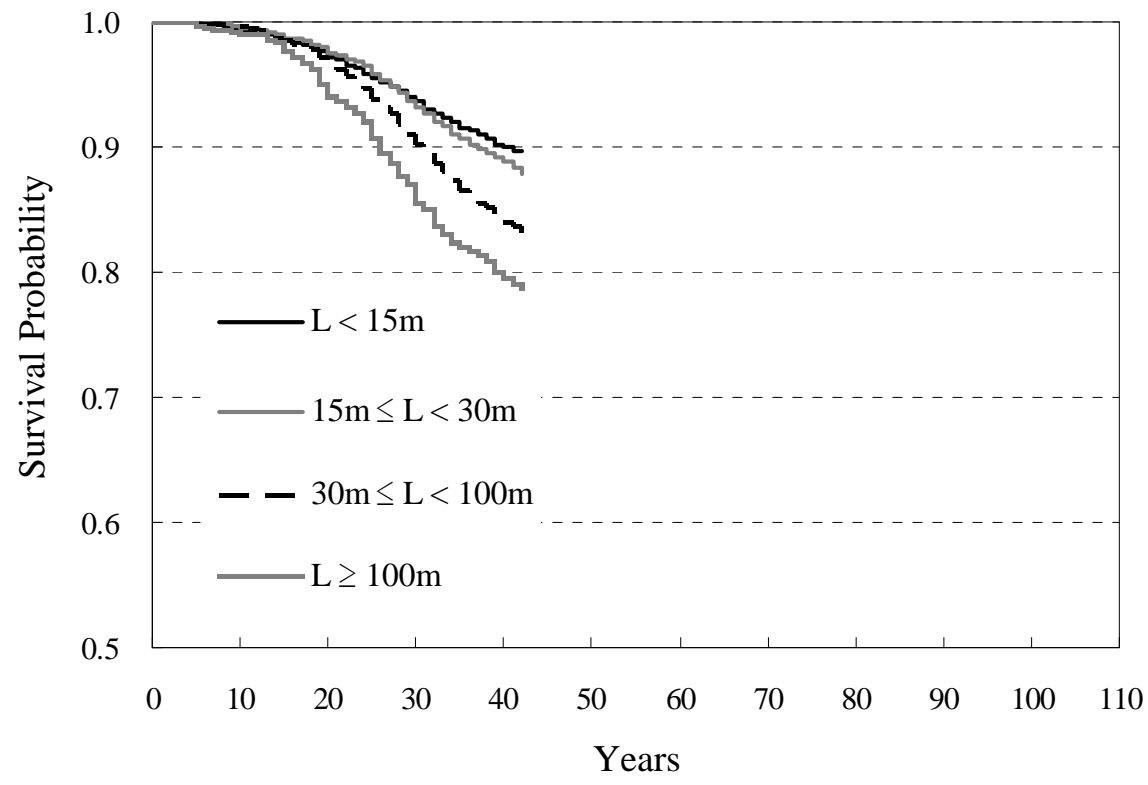

(g) Cohort 1960 


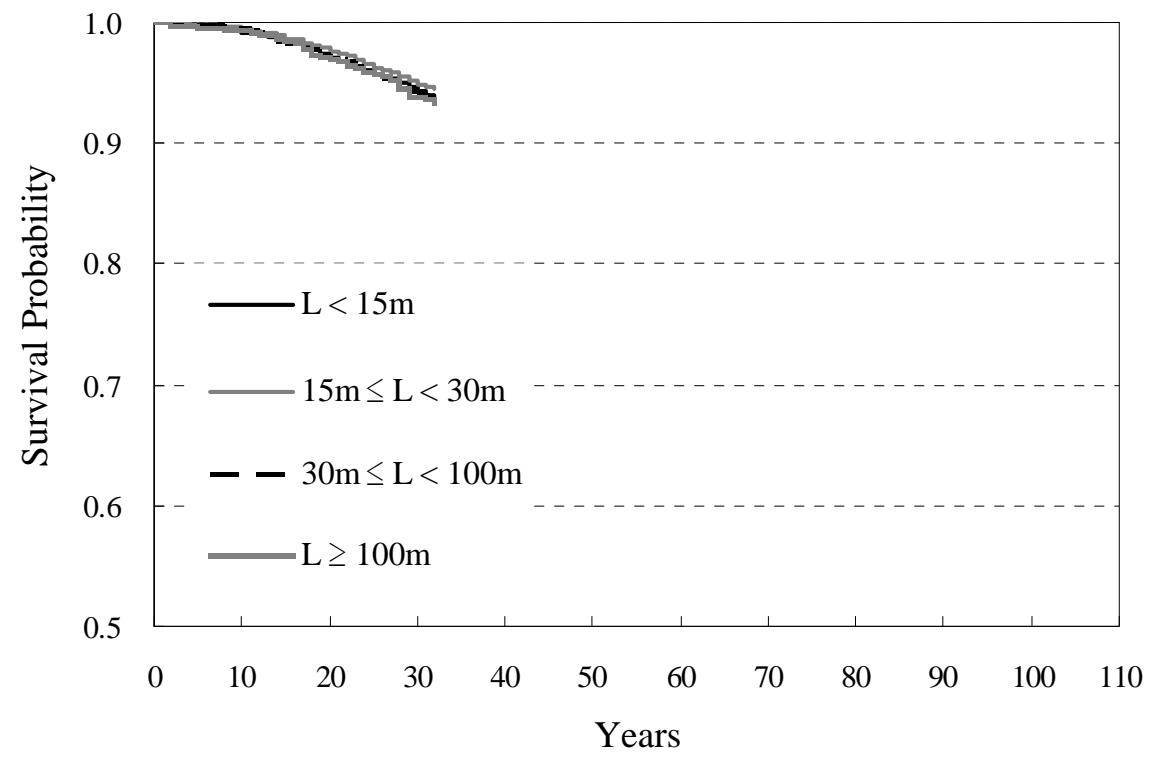

(h) Cohort 1970

Figure 12. Stratification with accordance to structure length 


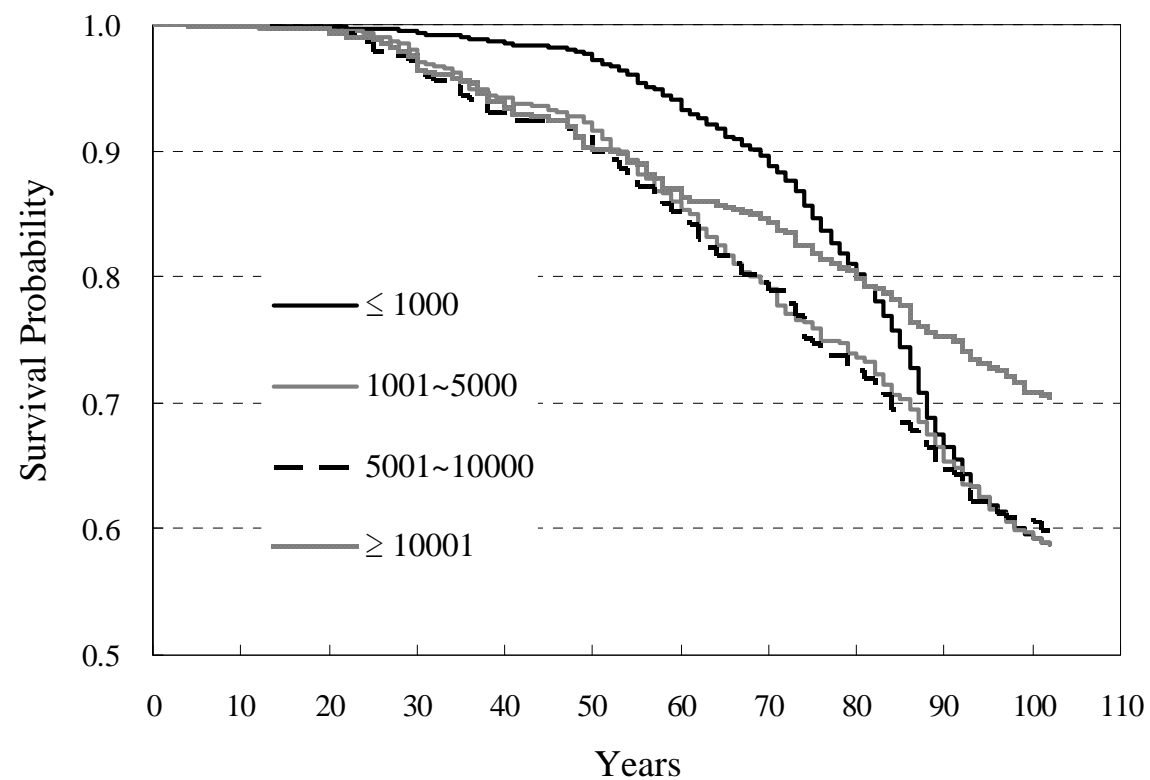

(a) Cohort 1900 


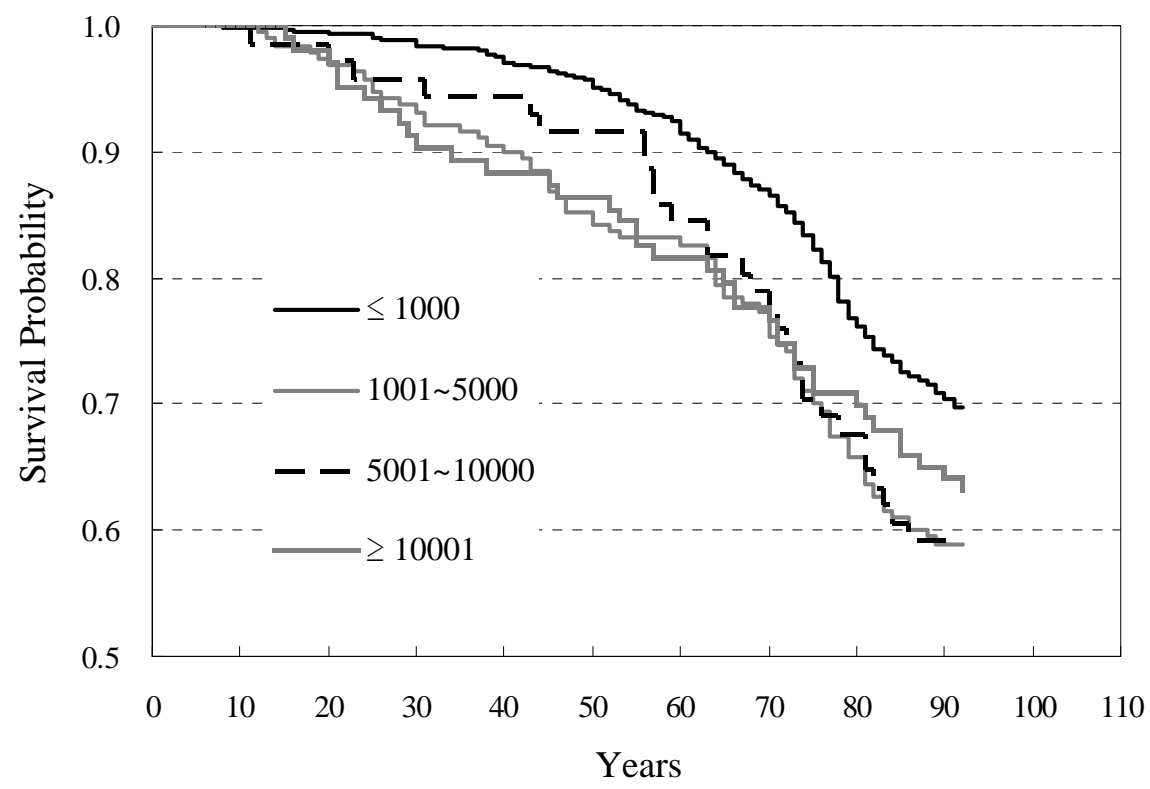

(b) Cohort 1910 


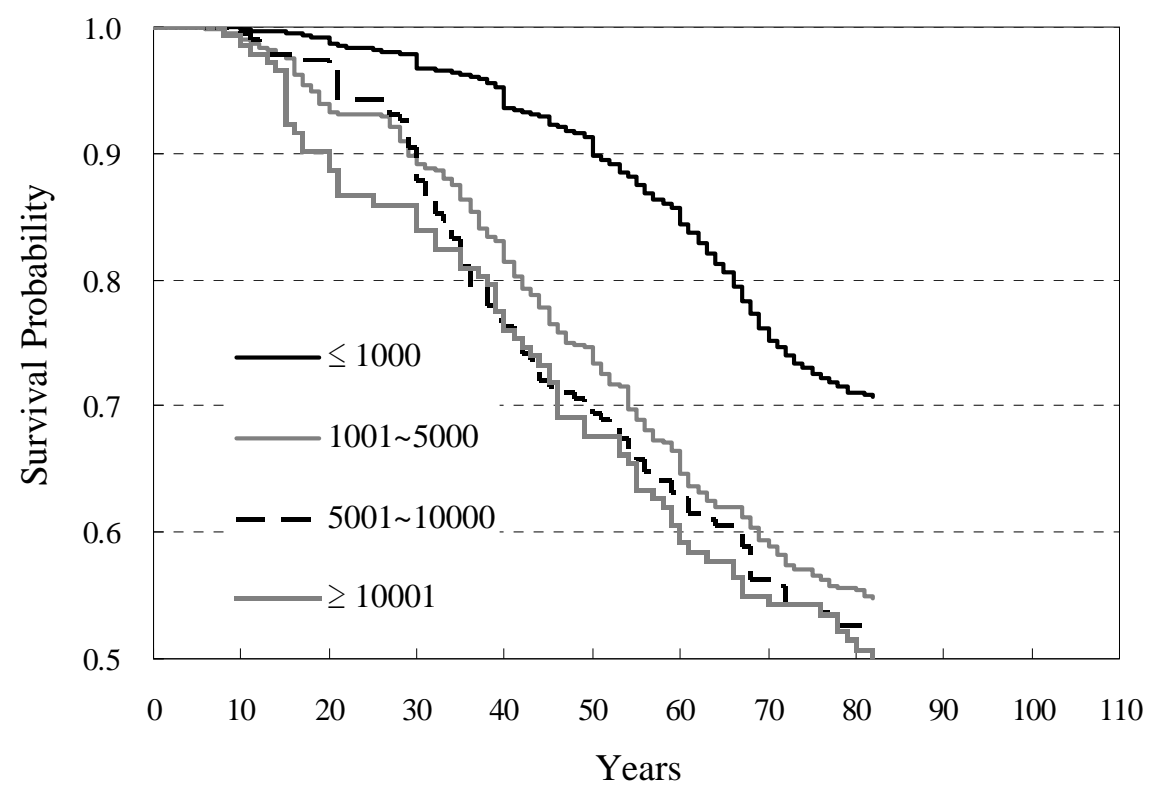

(c) Cohort 1920 


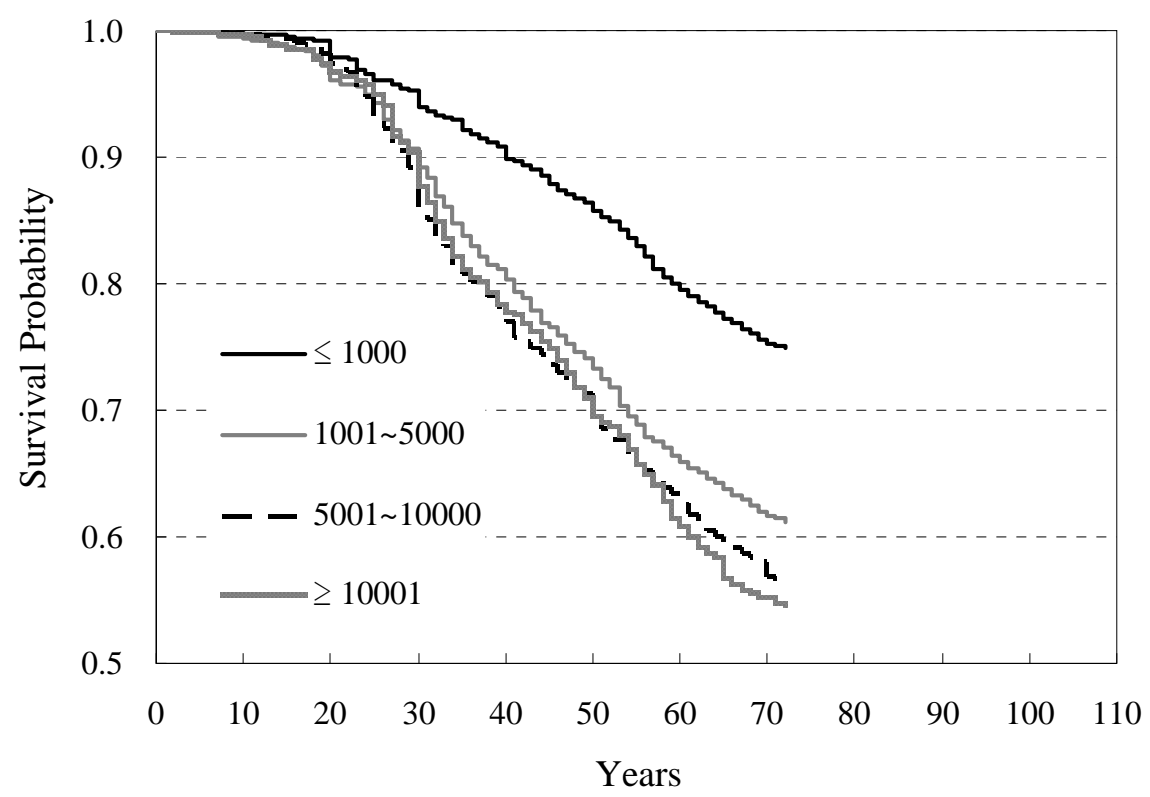

(d) Cohort 1930 


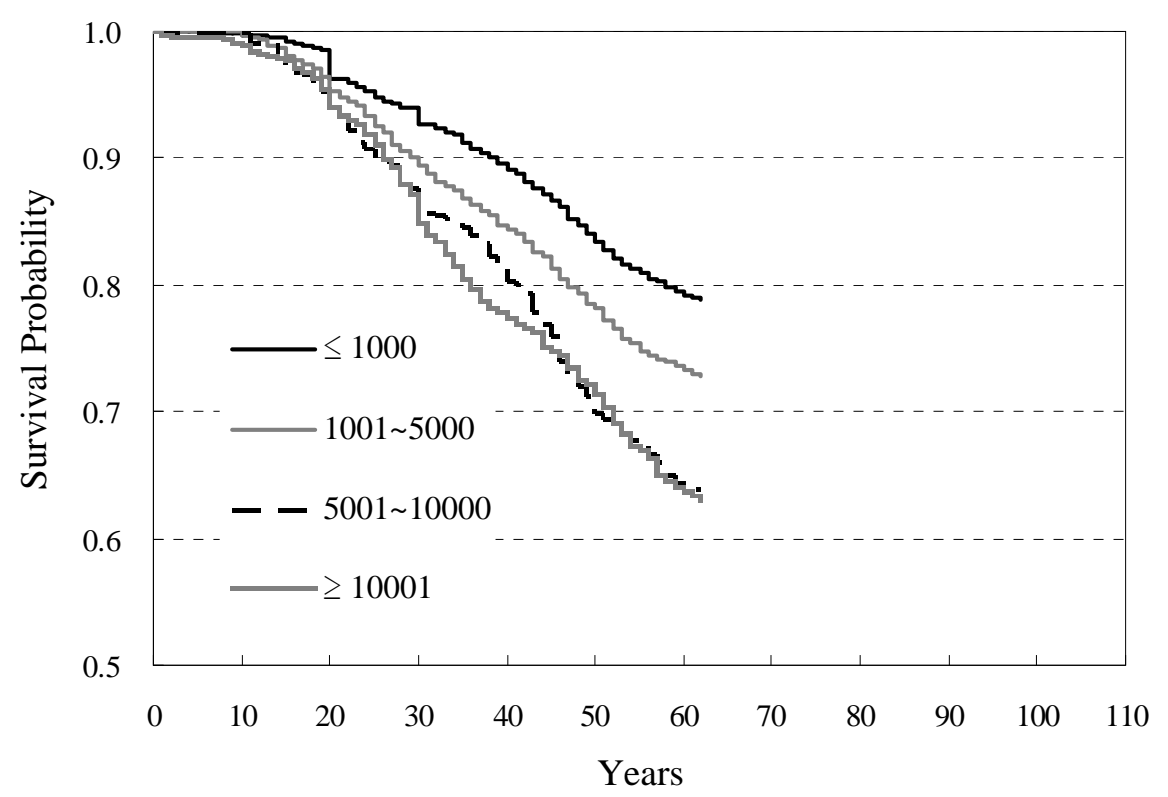

(e) Cohort 1940 


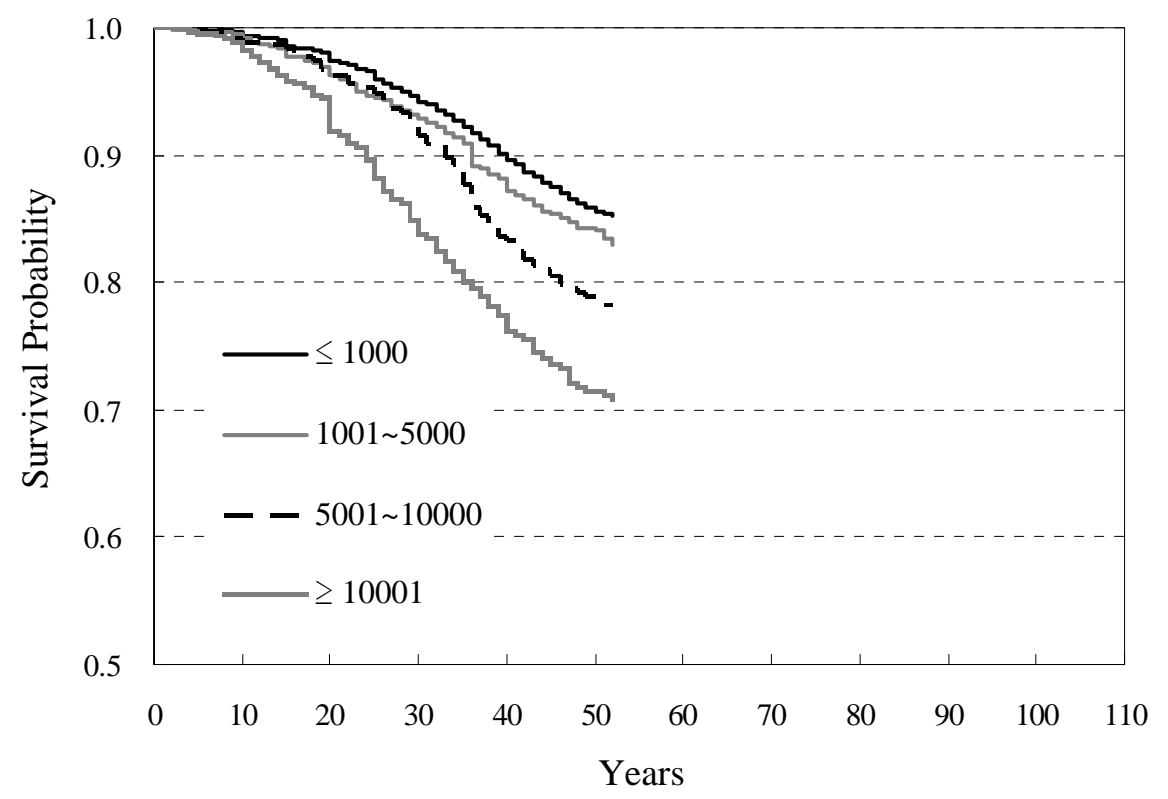

(f) Cohort 1950 


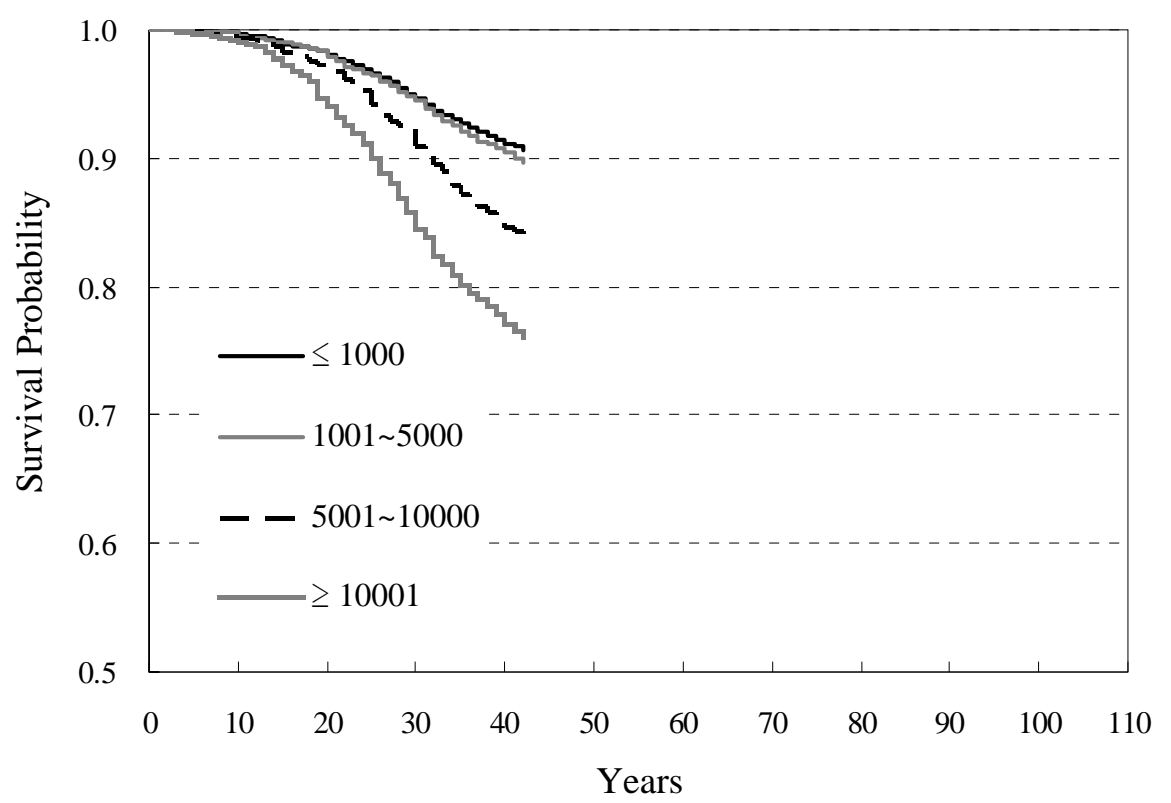

(g) Cohort 1960 


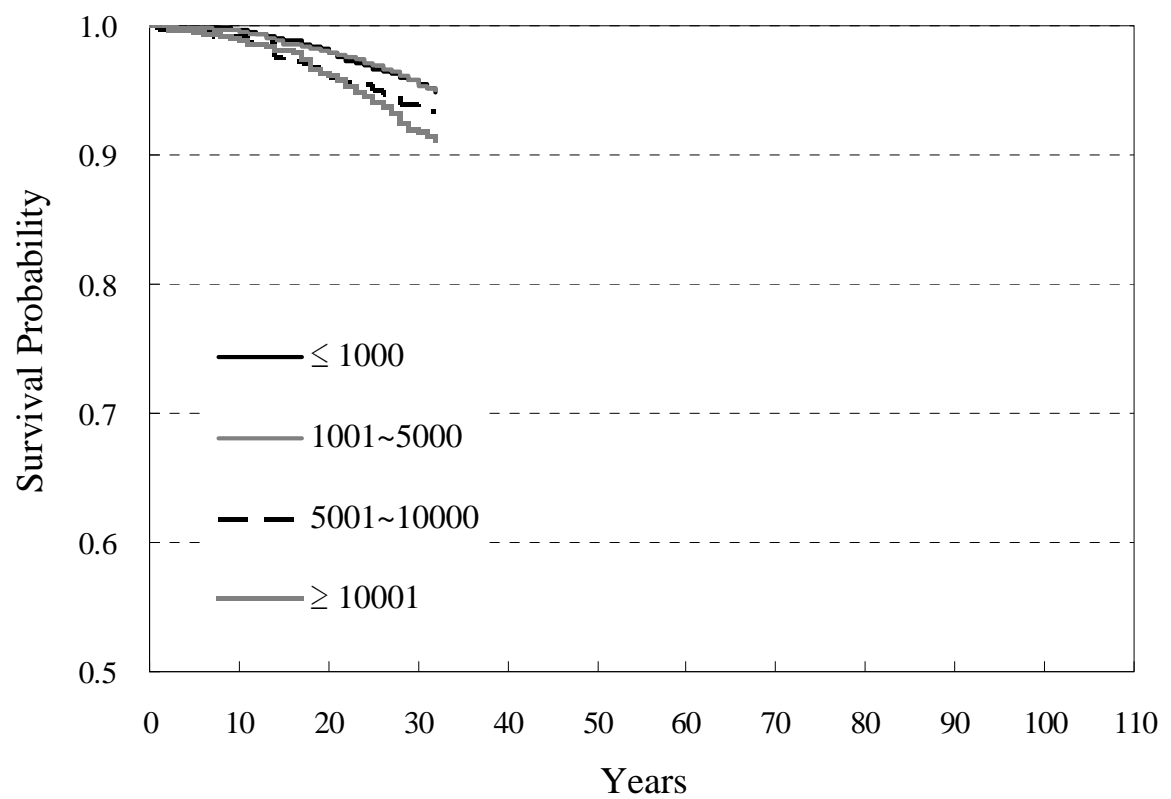

(h) Cohort 1970

Figure 13. Stratification with accordance to traffic volume 


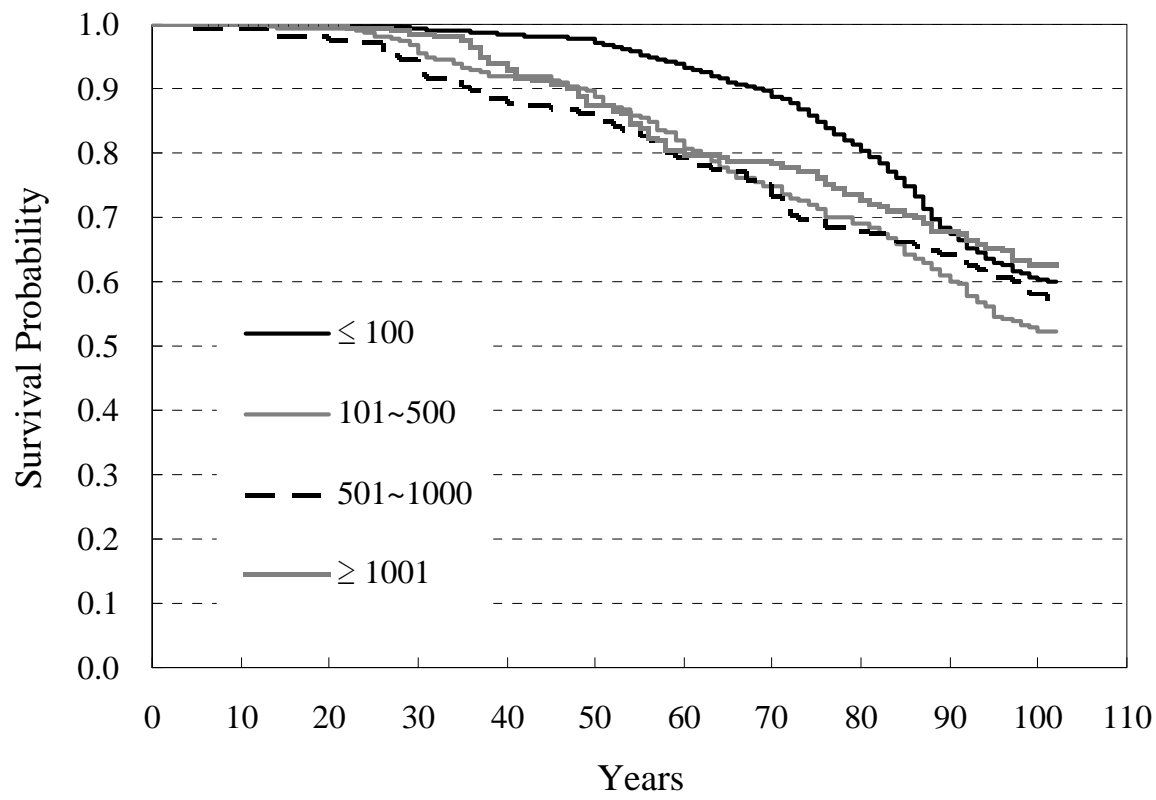

(a) Cohort 1900 


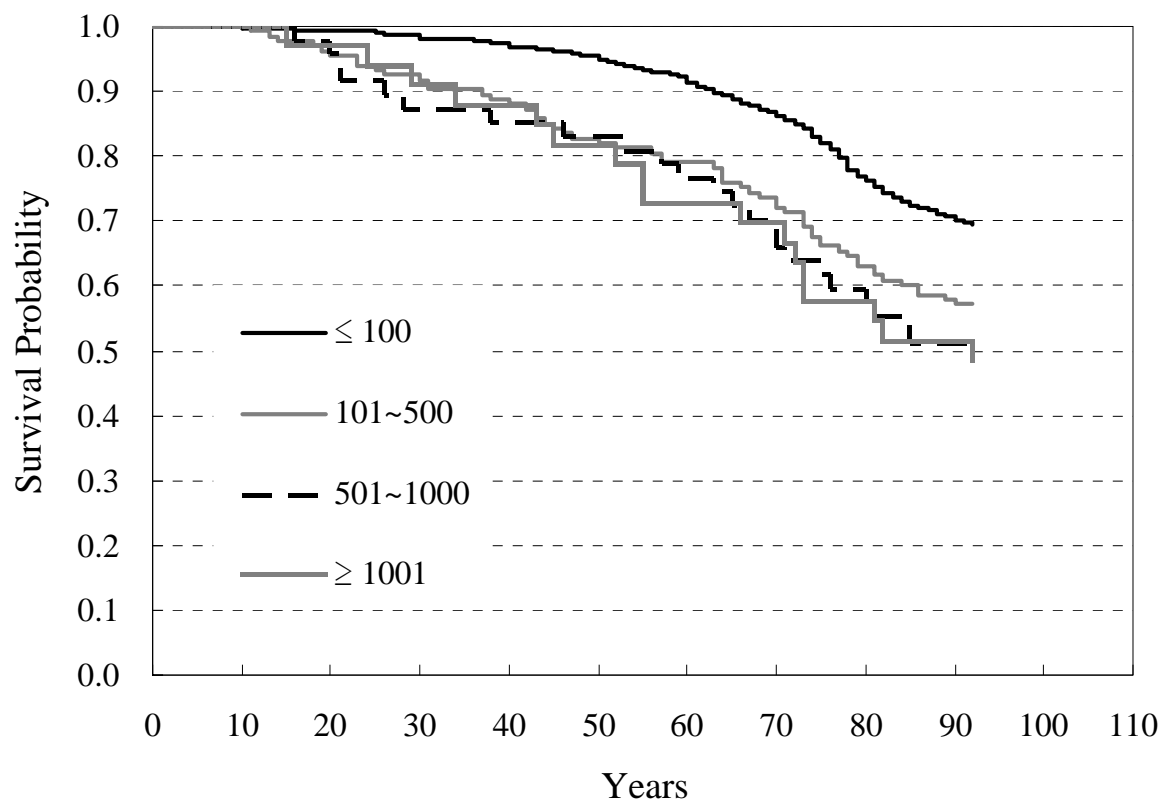

(b) Cohort 1910 


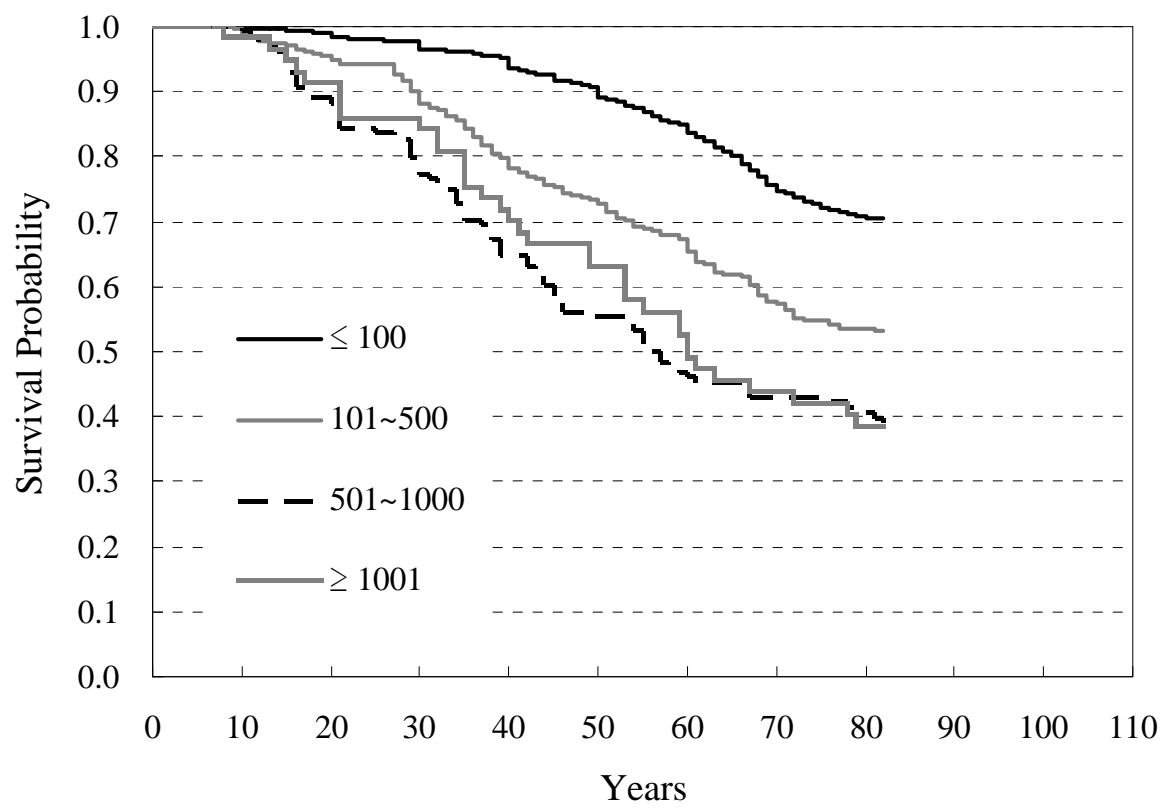

(c) Cohort 1920 


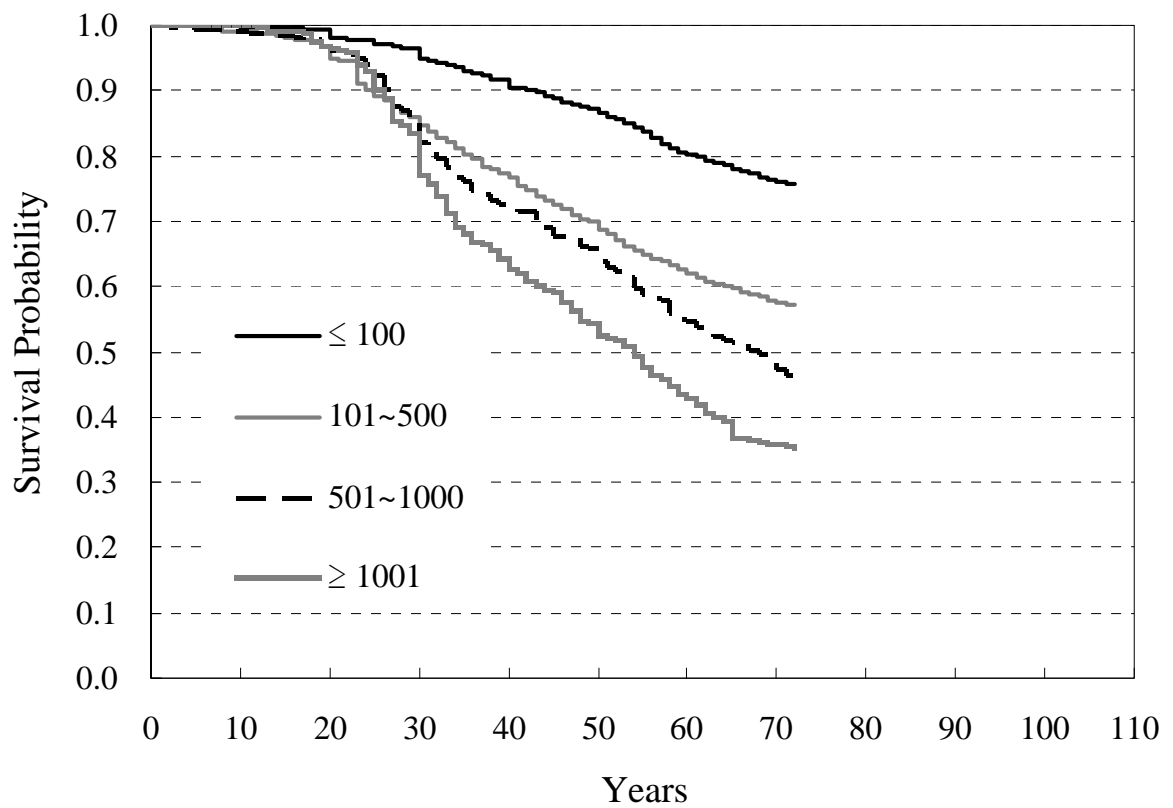

(d) Cohort 1930 


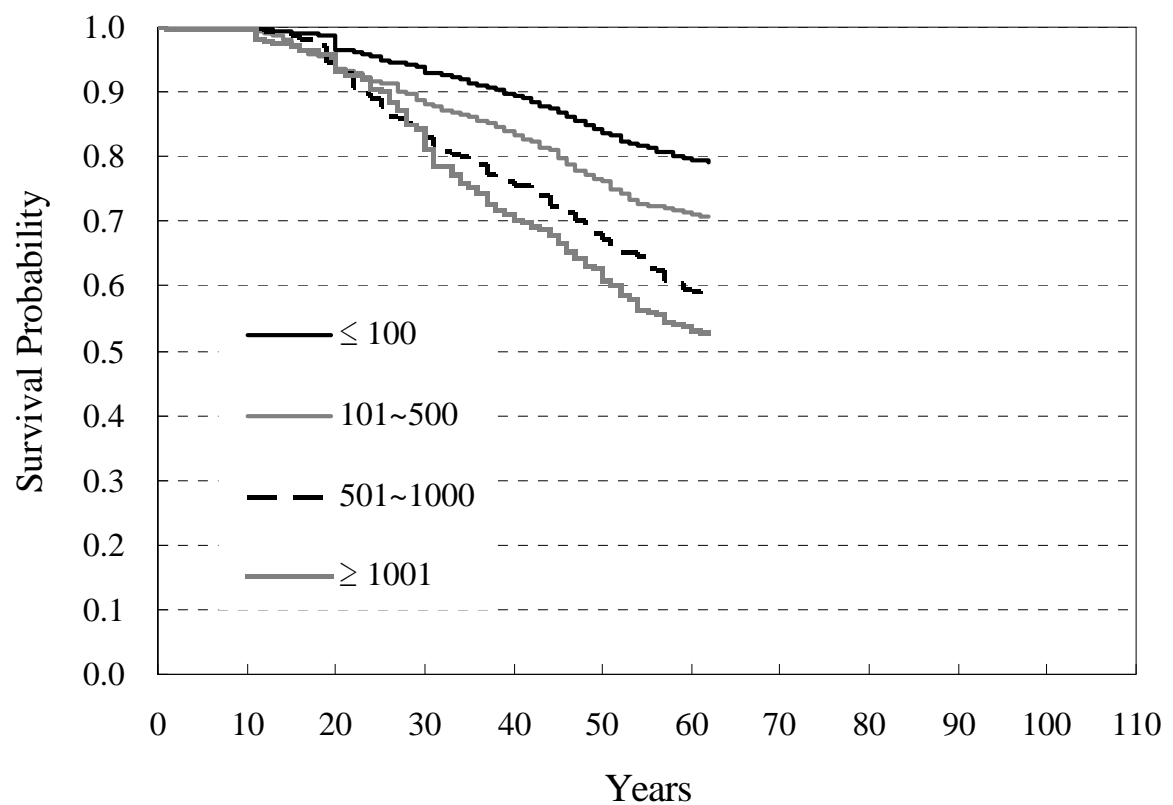

(e) Cohort 1940 


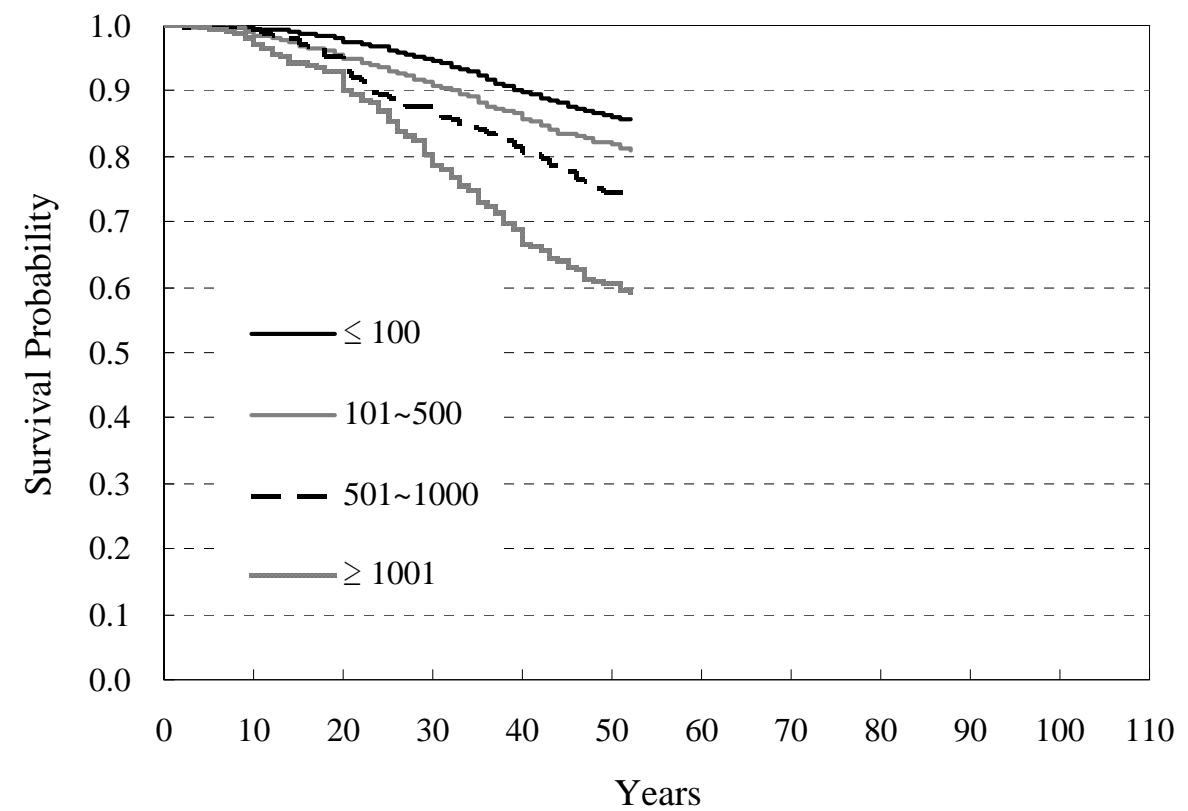

(f) Cohort 1950 


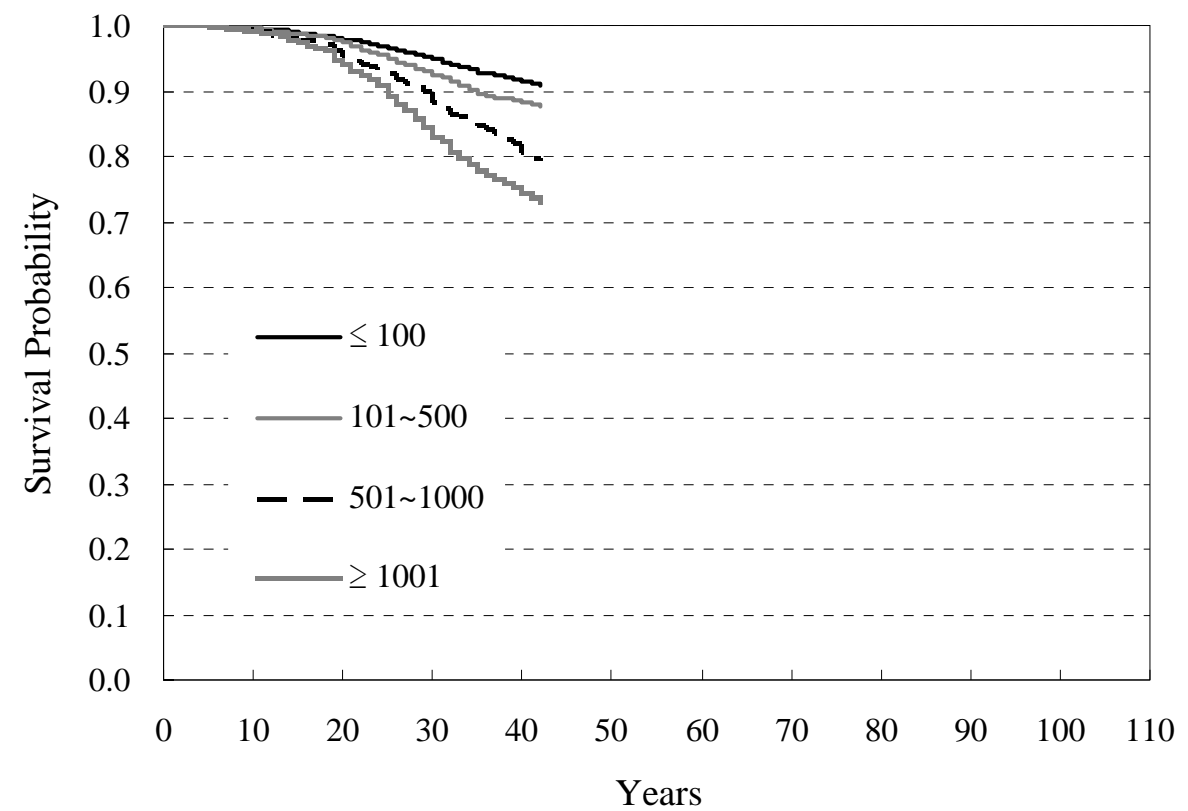

(g) Cohort 1960 


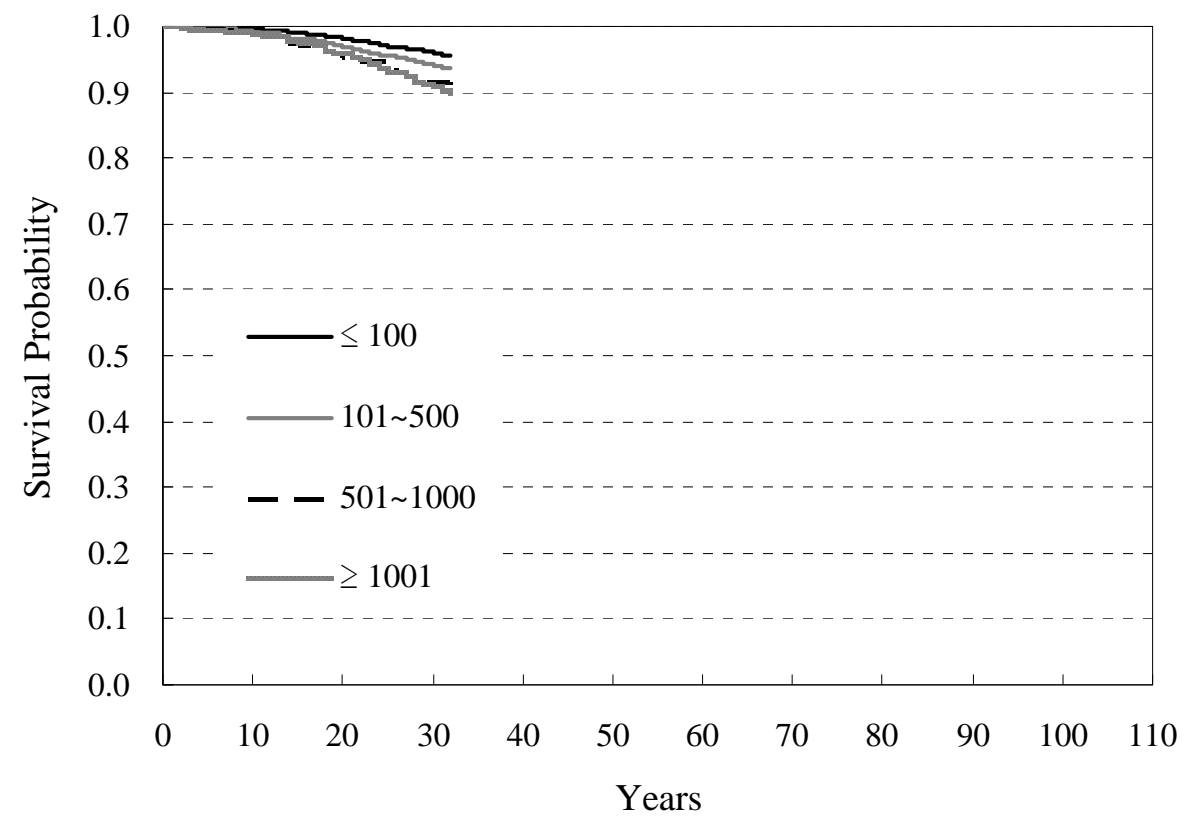

(h) Cohort 1970

Figure 14. Stratification with accordance to truck traffic volume 